\title{
Muséologies
}

Les cahiers d'études supérieures

muséologies

\section{Un cabinet d'égyptologue au Siècle des Lumières : le cabinet d'histoire naturelle du docteur Gagnon au Musée Stendhal de Grenoble (France)}

\section{Joëlle Rochas}

Volume 4, numéro 2, printemps 2010

URI : https://id.erudit.org/iderudit/1033538ar

DOI : https://doi.org/10.7202/1033538ar

Aller au sommaire du numéro

Éditeur(s)

Association Québécoise de Promotion des Recherches Étudiantes en Muséologie (AQPREM)

ISSN

1718-5181 (imprimé)

1929-7815 (numérique)

Découvrir la revue

Citer cet article

Rochas, J. (2010). Un cabinet d'égyptologue au Siècle des Lumières : le cabinet d'histoire naturelle du docteur Gagnon au Musée Stendhal de Grenoble (France). Muséologies, 4(2), 14-47. https://doi.org/10.7202/1033538ar
Résumé de l'article

Dans Vie de Henry Brulard, Stendhal évoque la maison grenobloise de son grand-père, demeure devenue depuis le Musée Stendhal. Il y décrit et dessine le cabinet d'histoire naturelle type du Siècle des Lumières, celui-là même qu'aurait constitué le docteur Gagnon, son aïeul. En 2008, à la maison Gagnon, on amorce la reconstitution du cabinet. Joëlle Rochas, experte scientifique attitrée à cette reconstitution, rend compte dans cet article des différentes étapes de ce projet et des divers problèmes qui ont dû être réglés entre son idéation et sa réalisation, tout en révélant sa portée historique et signifiante. 
Joëlle Rochas

\section{Un cabinet d'égyptologue au} Siècle des Lumières :

le cabinet d'histoire naturelle du docteur Gagnon au Musée Stendhal de Grenoble (France)

An meinen lieben Freund Bernhard Wolfmayr

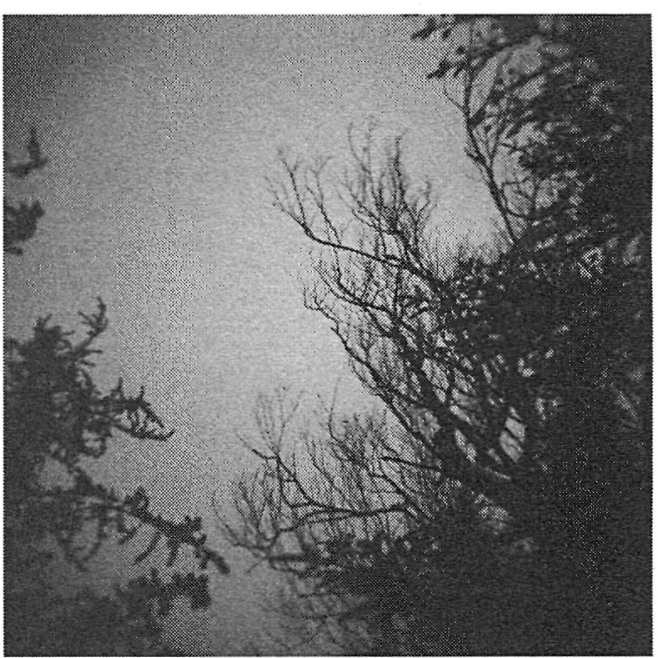




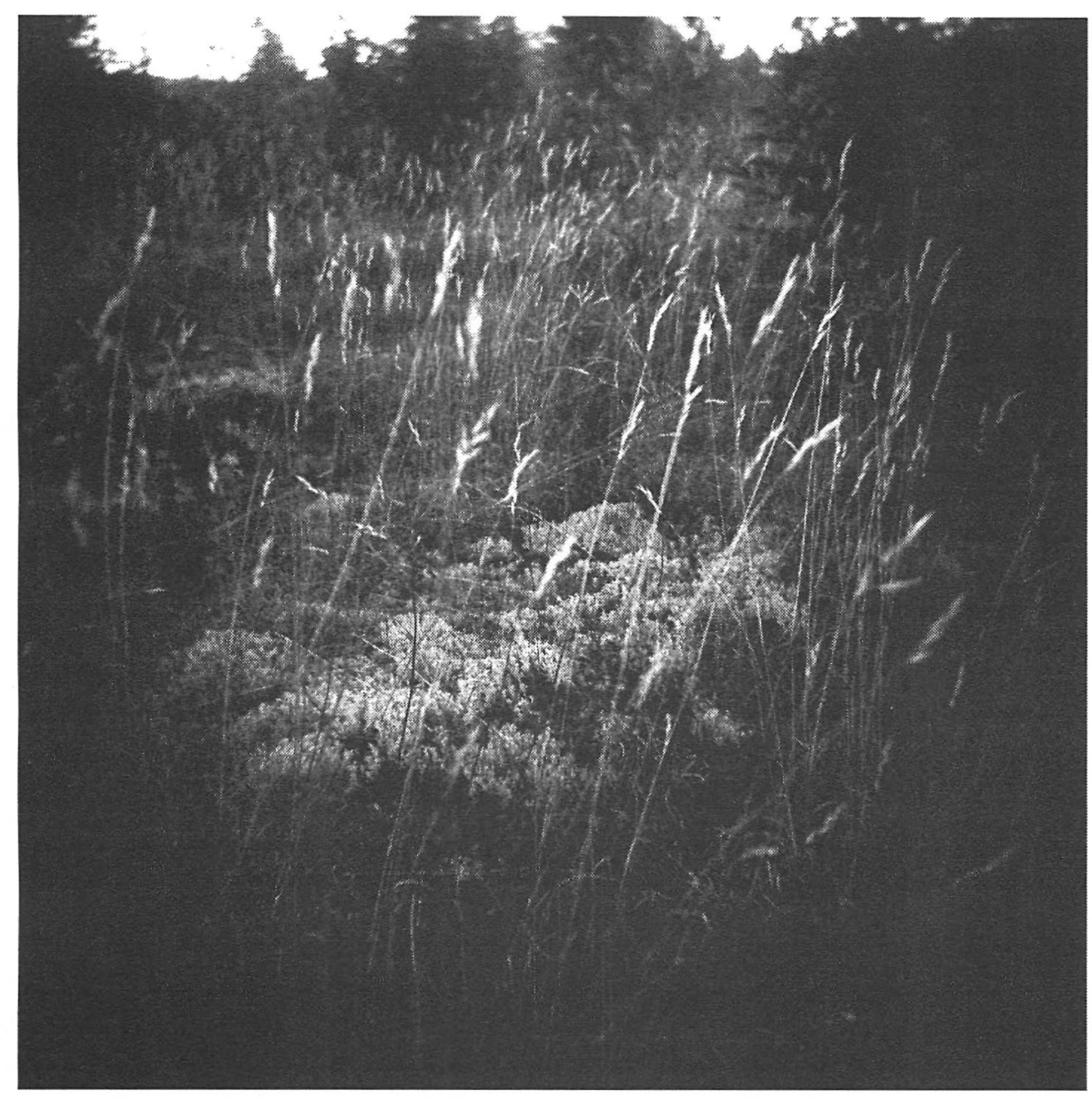

Joëlle Rochas est docteure en histoire, qualifiée en épistémologie - histoire des sciences. La thèse qu'elle a soutenue en 2006 à l'Université de Grenoble a pour titre: «Du cabinet de curiosités au muséum: les origines scientifiques du Muséum d'histoire naturelle de Grenoble (1773-1855) "'. Elle est actuellement bibliothécaire à I'Université de Savoie et chercheure associée au laboratoire CNRS EDYTEM (Environnements DYnamiques et TErritoires de Montagne). Elle poursuit ses travaux de recherche en histoire des sciences, principalement sur l'évolution de la curiosité du XVIe au XIXe siècle, en muséologie et sur le patrimoine des pays de l'arc alpin (France,
Autriche, Suisse, Allemagne, Italie, Slovénie). Elle associe ses travaux portant sur les cabinets de curiosités à ceux des chercheurs de l'Université de Poitiers ainsi qu'à ceux d'autres chercheurs en Europe, notamment pour le site européen $<$ Curiositas.org>, dédié aux cabinets de curiosités. La liste exhaustive de ses publications se trouve sur sa page Web (sur le site du laboratoire EDYTEM : <http://edytem.univ-savoie.fr/membres/rochas/rochas-joelle.htm>).

1

Un ouvrage adapté de la thèse a été publié en 2008 aux Éditions du Muséum de Grenoble : ROCHAS, Joëlle. Muséum de Grenoble : une histoire naturelle. Grenoble: Éditions du Muséum, 2008, 273 p. 


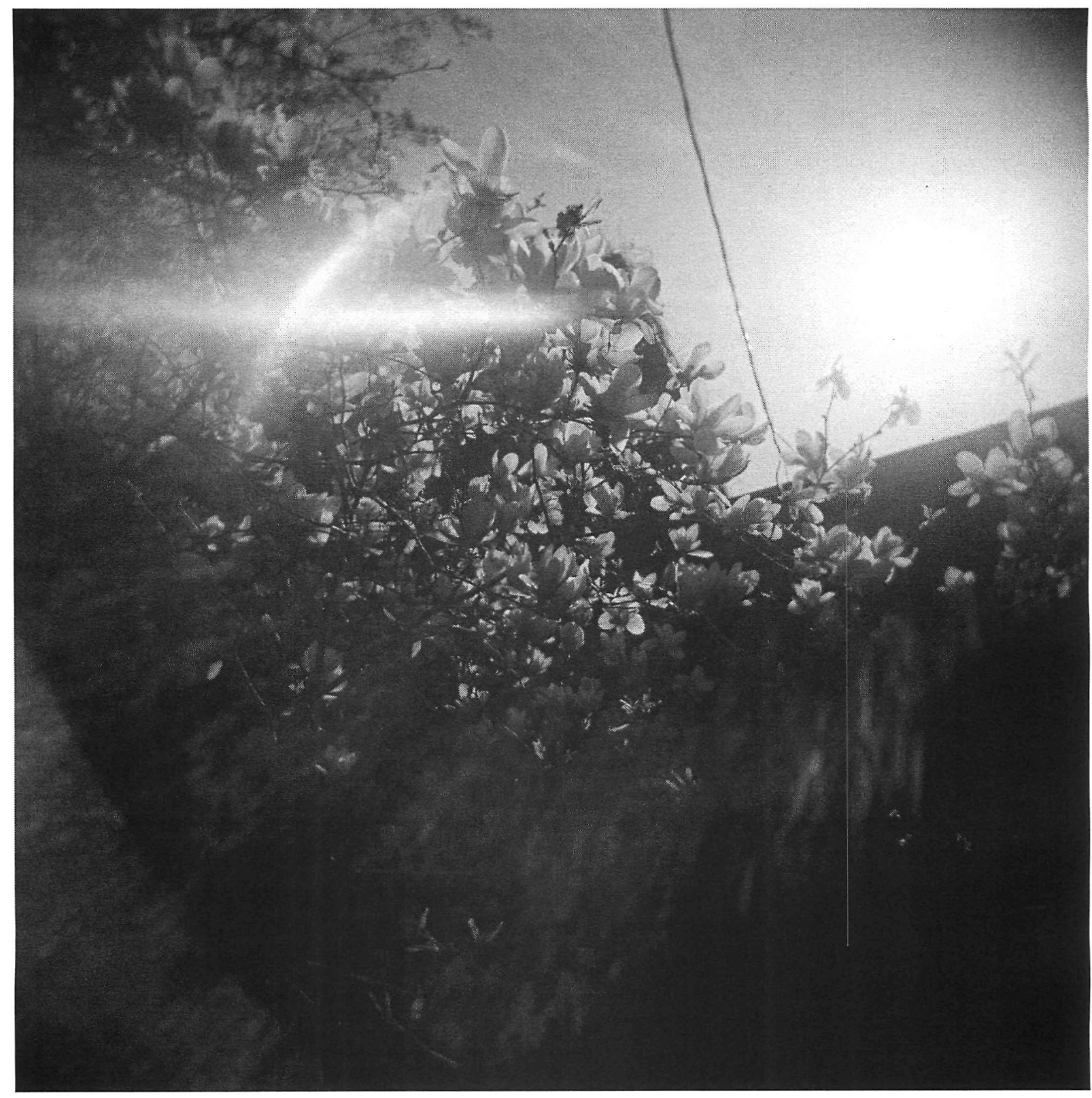


Dans Vie de Henry Brulard, Stendhal évoque la maison grenobloise de son grand-père, demeure devenue depuis le Musée Stendhal. Il y décrit et dessine le cabinet d'histoire naturelle type du Siècle des Lumières, celui-là même qu'aurait constitué le docteur Gagnon, son aïeul. En 2008, à la maison Gagnon, on amorce la reconstitution du cabinet. Joëlle Rochas, experte scientifique attitrée à cette reconstitution, rend compte dans cet article des différentes étapes de ce projet et des divers problèmes qui ont dû être réglés entre son idéation et sa réalisation, tout en révélant sa portée historique et signifiante. 
Illustration 1

Portrait de Stendhal par Pierre-Joseph Dedreux-Dorcy, huile sur toile.

Collection Musée Stendhal de Grenoble, $n^{\circ} 830$

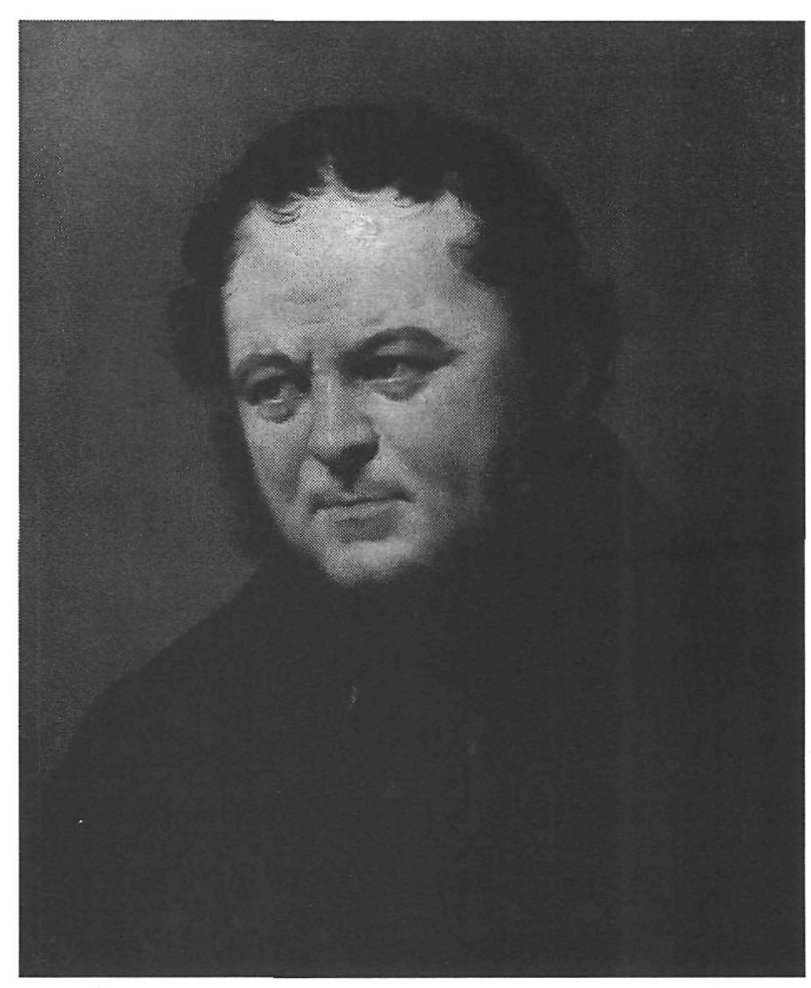

La Ville de Grenoble dans les Alpes françaises a entrepris en 2008 la restauration de la maison du docteur Henri Gagnon, grand-père de l'écrivain Stendhal, un des plus grands écrivains français, connu aussi bien sur le plan national qu'international. Le docteur Gagnon, véritable esprit des Lumières, eut une influence considérable sur la formation de son petit-fils dont il assura en partie l'éducation. Personnage stendhalien par excellence, le docteur Gagnon est au cœur du roman autobiographique de Stendhal, Vie de Henry Brulard, où l'auteur évoque les premières années de sa jeunesse dans la maison de son grand-père à Grenoble. Stendhal nous dit que son grand-père possédait à la fin du XVIII ${ }^{\mathrm{e}}$ siècle un cabinet d'histoire naturelle dans sa maison à Grenoble. Il en dessine le plan.

Historienne des sciences, auteur en 2006 d'une thèse sur le Muséum de Grenoble et spécialiste des cabinets de curiosités et des cabinets d'histoire naturelle, j'ai été sollicitée par la direction des Affaires culturelles de la Ville de Grenoble pour la reconstitution du cabinet d'histoire naturelle du docteur Gagnon au sein du Musée Stendhal. J'ai également été mandatée à titre d'experte scientifique pour recréer, dans le prolongement du cabinet d'his- 
toire naturelle, la terrasse du docteur Gagnon, terrasse communément appelée à Grenoble "la Treille de Stendhal ". L'ouverture au public de la maison Gagnon et de l'ensemble du Musée Stendhal devrait coïncider avec les Journées européennes du Patrimoine en septembre 2011. Le nouveau Musée Stendhal permettra ainsi de découvrir, à l'intérieur d'une maison du XVIII ${ }^{\mathrm{e}}$ siècle et voisinant un grand salon à l'italienne et une bibliothèque, un cabinet d'histoire naturelle et un jardin d'expérimentation chers aux amateurs d'histoire naturelle à l'époque des Lumières.

Le présent article a pour but de présenter ce qu'était un cabinet d'histoire naturelle à l'époque des Lumières, tout en relatant les différentes phases d'une reconstitution dans une maison d'écrivain. Il saura faire la part entre la réalisation idéale et la réalisation possible, entre la rigueur scientifique et les exigences du maître d'œuvre, entre la vérité historique et les contraintes muséographiques. L'expérience n'en demeure pas moins riche, car cette reconstitution porte témoignage sur le caractère fort original des cabinets grenoblois de l'époque. En effet, dans l'immense mouvement des sciences où l'on évolue au XVIII ${ }^{\mathrm{e}}$ siècle de cabinets de curiosités en cabinets d'histoire naturelle, les cabinets grenoblois ont pour originalité d'être des cabinets d'histoire naturelle encore très influencés par les cabinets de curiosités. Les curieux qui rassemblent ces collections s'intéressent à l'égyptologie, aux collections exotiques. Leurs cabinets ne sont en rien archaïques, mais plutôt précurseurs dans la mesure où ils annoncent déjà, avec leur intérêt pour les collections anthropologiques, la phase ultérieure de la métamorphose des musées d'histoire naturelle, l'avènement prochain des muséums. La reconstitution du cabinet d'histoire naturelle de Gagnon sera l'unique témoignage de ces cabinets grenoblois se reconnaissant de la double appartenance. Il portera notamment témoignage sur l'égyptologie à laquelle s'intéressent très tôt les Grenoblois et dont se ressentent leurs collections. Il annoncera l'arrivée du jeune Champollion, ami du docteur Gagnon, égyptologue, futur découvreur des hiéroglyphes, un des premiers gardes du futur Muséum de Grenoble et le premier savant à avoir étudié les collections égyptiennes des cabinets de curiosités dauphinois. grand-père maternel, le docteur Gagnon, un médecin réputé 


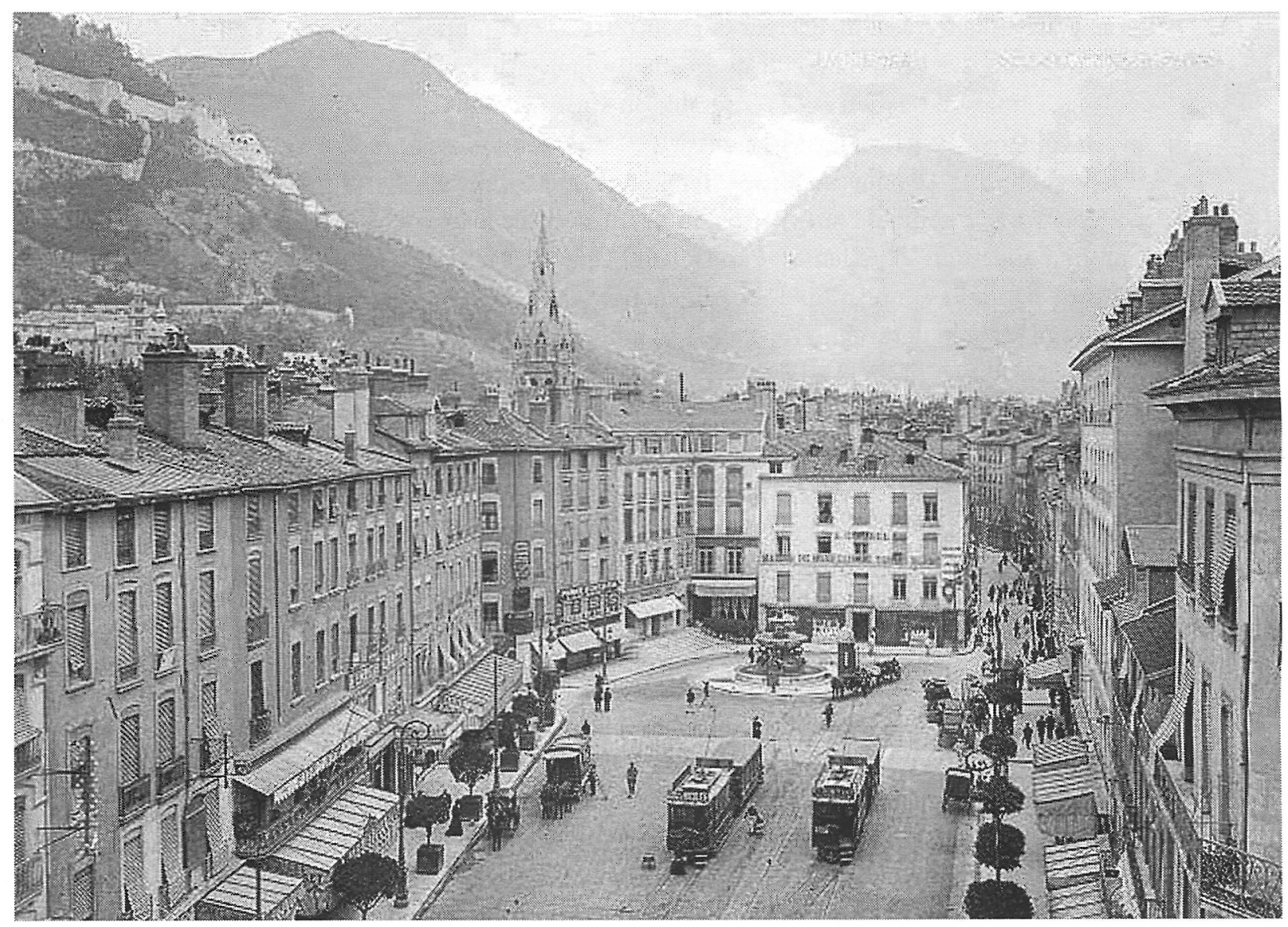

Illustration 2

Vue ancienne de Grenoble au début du XX $\mathrm{XX}^{\mathrm{e}}$ siècle. La place Grenette; au fond, derrière la fontaine, la maison du $\mathrm{D}^{\mathrm{r}}$ Gagnon avec sa façade blanche. Illustration tirée de Gallica, bibliothèque numérique de la Bibliothèque nationale de France, que nous devons à Gérald Rannaud, membre du comité scientifique du Musée Stendhal 
de la ville. Stendhal aimait son grand-père auquel il doit sa formation. Son œuvre toute entière se ressent de cette influence. C'est dans son roman Vie de Henry Brulard que l'auteur nous raconte son enfance à Grenoble. Il évoque la maison de son grandpère et, parmi les nombreuses pièces que celle-ci comporte, le cabinet d'histoire naturelle, cabinet typique de la fin du XVIII ${ }^{\mathrm{e}}$ siècle, avec ses armoires en bois blanc remplies de spécimens d'histoire naturelle. Le cabinet est prolongé par une terrasse ornée de vigne et de fleurs, véritable laboratoire de l'amateur de botanique du XVIII ${ }^{e}$ siècle. Stendhal dessine le plan du cabinet avec cette légende brève : "Cabinet d'histoire naturelle. Minéraux, oiseaux....".

Et c'est sur cette brève évocation qu'est né, à Grenoble, le projet de reconstitution du cabinet d'histoire naturelle du grand-père de Stendhal. Pari fou quand on pense aux quelques traces que nous a laissées Stendhal, mais reconstitution possible grâce aux études menées sur les cabinets dans les appartements grenoblois du XVIII ${ }^{\mathrm{e}}$ siècle.

Grenoble est aujourd'hui une ville de 160000 habitants, située dans les Alpes françaises. C'est une ville d'ingénieurs qui a su développer son potentiel industriel tout au long du $\mathrm{XX}^{\mathrm{e}}$ siècle. Elle accueille en ce début de XXI ${ }^{\mathrm{e}}$ siècle des activités d'enseignement et de recherche, et ses nombreux laboratoires font d'elle une métropole scientifique et technologique internationale. S'illustrant dès le XVIII ${ }^{\mathrm{e}}$ siècle dans le domaine des lettres puis au XIX ${ }^{\mathrm{e}}$ siècle dans celui des techniques, la ville a vu naître ou grandir des hommes illustres, savants, philosophes ou musiciens, que Paris ou la scène internationale vont révéler. On peut ainsi citer en relation avec notre propos l'égyptologue Jean-François Champollion (1790-1832), le philosophe et écrivain Étienne Bonnot (1715-1780), plus connu sous le nom de Condillac, le musicien Hector Berlioz (1803-1869) et, bien sûr, Henri Beyle dit Stendhal (1783-1842). Rappelons que c'est à Grenoble qu'ont eu lieu en 1788 des événements de protestation contre l'autorité royale qui sont analysés par les historiens comme l'amorce de la Révolution française. Les notables de Grenoble, dont le docteur Gagnon, s'étaient en effet réunis en assemblée et avaient réclamé la réunion des États généraux ${ }^{3}$.

Très attachée à la valorisation du patrimoine stendhalien, la Ville de Grenoble a déjà entrepris en 2002 la restauration de l'appartement
2

STENDHAL. Vie de Henry Brulard. Paris: Gallimard, 1973, p. 180. (Cette édition contient la reproduction des croquis de Stendhal.) 3

Le Musée de la Révolution française se situe à Vizille, ville du sud de la région grenobloise où se tint cette première assemblée. 


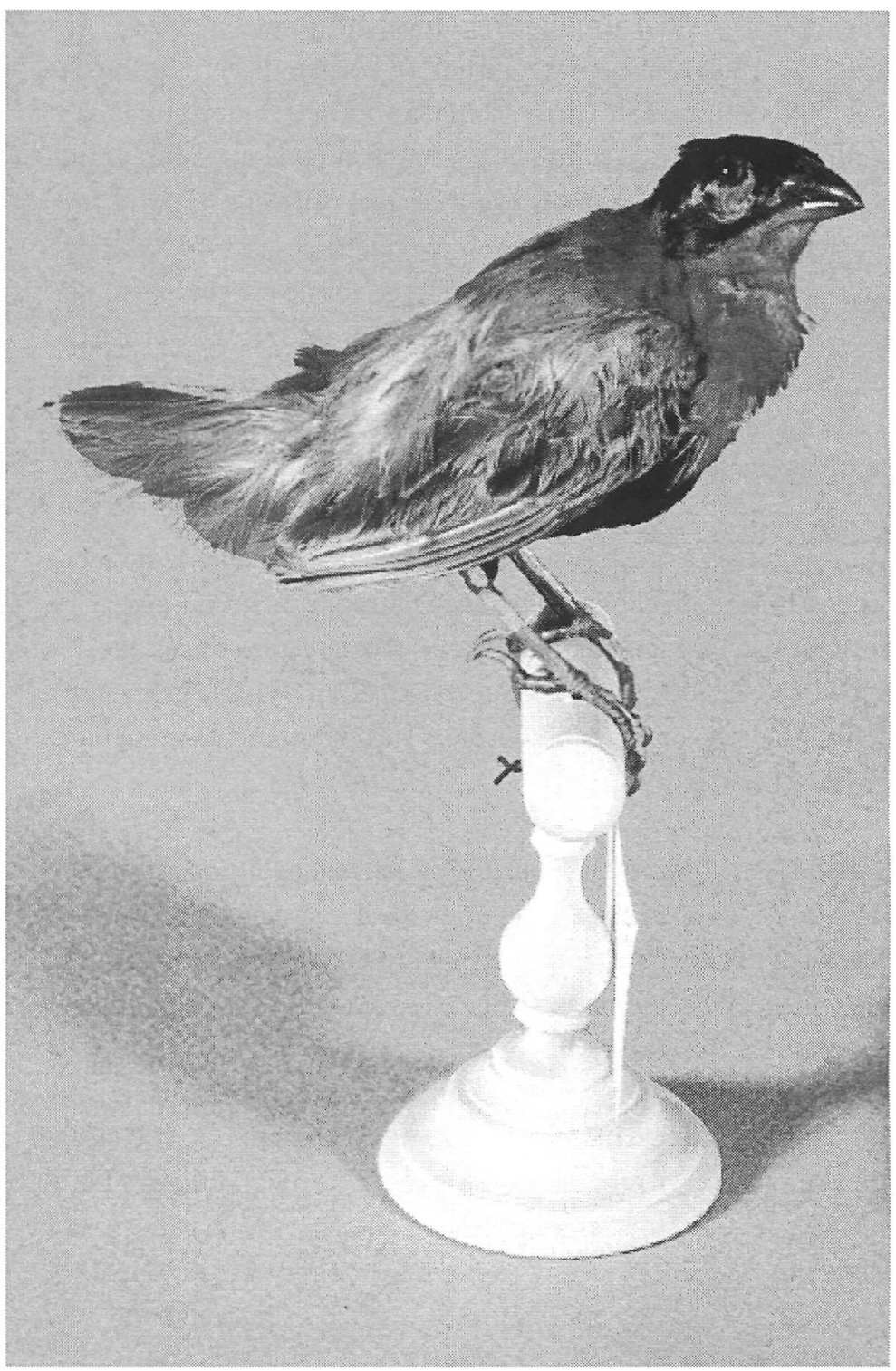

Illustration 3

Oiseau illustrant les collections ornithologiques exotiques du cabinet de curiosités des Antonins.

Collection du Muséum d'histoire naturelle de Grenoble 
natal de Stendhal, suivie en 2008 de celle de la maison Gagnon, maison du grand-père dans laquelle l'écrivain a grandi et où figure le cabinet à reconstituer. Cette seconde restauration marque une étape dans la création d'une entité plus globale, le futur Musée Stendhal, lequel rassemblera l'ensemble du patrimoine stendhalien à Grenoble. Nous reviendrons au cours de cet article sur ce concept englobant choisi pour l'entité "Musée Stendhal ". La Ville conçoit déjà ce musée comme « un atout de politique majeur", par ses dimensions culturelle, touristique et économique. Mais le projet répond aussi à deux exigences, l'une intérieure, venant des Grenoblois eux-mêmes et de l'intérêt qu'ils vouent à leur écrivain ainsi qu'à son œuvre (il y a là un phénomène d'identification des Grenoblois à Stendhal, une véritable reconnaissance et une appropriation de son œuvre), l'autre extérieure, venant de l'étranger où l'on connaît Grenoble par l'intermédiaire de ses deux personnages emblématiques : Berlioz et Stendhal. La maison natale d'Hector Berlioz ayant été récemment restaurée par le Conseil général de l'Isère ${ }^{4}$, la Ville de Grenoble s'est engagée à son tour dans le projet du Musée Stendhal. Le nouveau Musée Stendhal devrait être inauguré en septembre 2011 à l'occasion des Journées européennes du Patrimoine.

\section{Le docteur Gagnon, grand-père de Stendhal}

Le docteur Gagnon est un personnage incontournable dans le Grenoble de la fin du XVIII ${ }^{\mathrm{e}}$ siècle. Bibliothèque, Cabinet d'histoire naturelle, Académie delphinale ${ }^{5}$, École de chirurgie, École centrale, il participe à toutes les nouvelles institutions littéraires, artistiques ou scientifiques dont se dote la ville à la veille de la Révolution et jusqu'à l'Empire. Passionné de sciences naturelles, il est l'auteur d'un mémoire à l'origine de la création en 1773 du Cabinet d'histoire naturelle de Grenoble, ancêtre direct de l'actuel Muséum de Grenoble:

À peine avait-on réalisé le projet d'une bibliothèque publique qu'on commence à éprouver les bons effets que doit produire cet établissement : le vœu unanime de tous les citoyens [est de former] un cabinet de physique [et] d'histoire naturelle. Déjà, des personnes en place et des gens de lettres ont voulu concourir à en rassembler les matériaux : une ample collection
4

Le Musée départemental Berlioz se situe à La Côte Saint-André (en Isère), ville natale de Berlioz et lieu du Festival Berlioz, événement annuel de renommée internationale. La recomposition de l'aménagement intérieur de la maison natale du musicien a été réalisée d'après les descriptions laissées par son père, le docteur Berlioz, et de ce que connaissent les conservateurs du patrimoine des intérieurs bourgeois d'une petite ville dauphinoise au début du XIX siècle. Précisons également ici que le musée Maison Champollion, résidence familiale des Champollion à Vif (en Isère), autre musée départemental, est quant à lui en cours de restauration et qu'il rouvrira ses portes en 2010.

5

C'est le nom de l'académie fondée à la fin du XVIIIle siècle dans l'ancienne province du Dauphiné dont Grenoble était la capitale. 
Illustration 4

Portrait du docteur Henri Gagnon. Reproduction photographique du portrait à l'huile déposé au Musée Stendhal, Grenoble

6

CAGNON, Henri. "Mémoire pour la création du Cabinet de physique et d'histoire naturelle". In. Délibération de la Bibliothèque de Crenoble du 12 juillet 1773: document $n^{\circ} 9$ (Bibliothèque municipale [d'études et d'information] de Crenoble/BMG, R 8709).

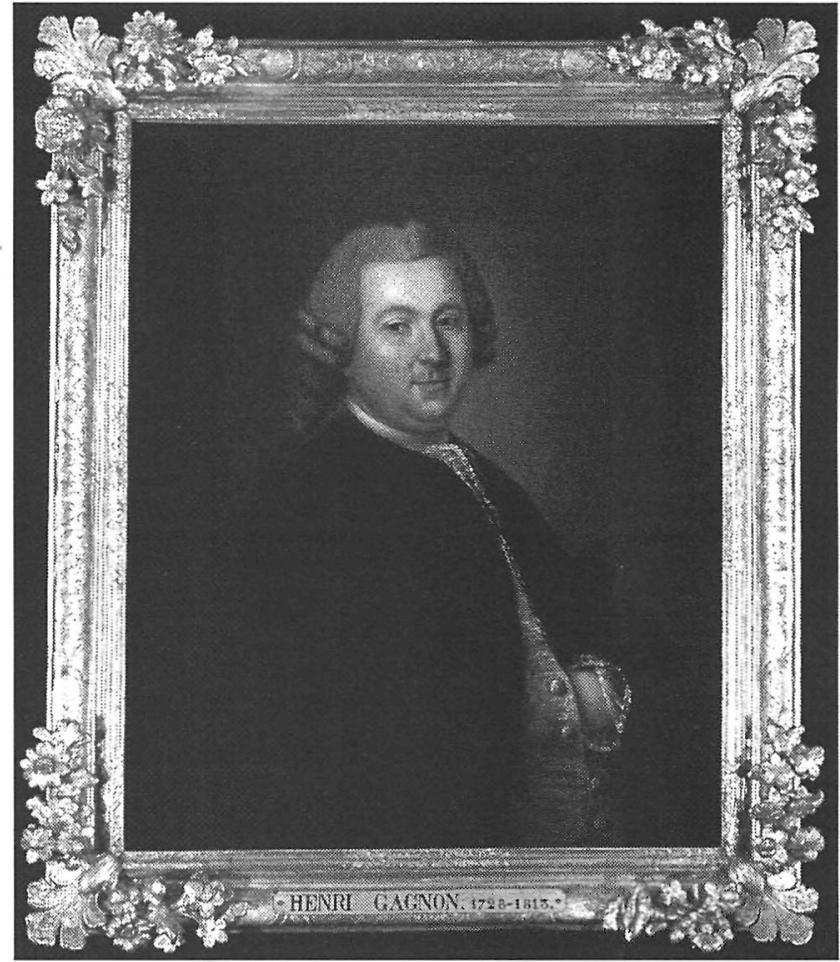

de minéraux par eux rassemblés avec autant de zèle que d'intelligence des parties isolées des trois règnes, des instruments de physique, comme des cabinets particuliers, n'attendent qu'un dépôt public pour former un établissement qui, avec la bibliothèque, coopérera à la révolution si nécessaire au progrès des sciences ${ }^{6}$.

Il fera partie du premier groupe des directeurs qui créera la bibliothèque de Grenoble et c'est lui qui procèdera à la constitution des premières collections du Cabinet d'histoire naturelle de Grenoble. On lui doit ainsi le premier spécimen de zoologie exotique de l'actuel Muséum de Grenoble (un veau marin acquis en 1779).

Mais on lui doit surtout le transport du cabinet de curiosités ainsi que de la bibliothèque de l'abbaye de Saint-Antoine que la ville reçoit en don de l'Ordre des Antonins au moment de sa dissolution en 1777 . Ce cabinet transporté par Gagnon va constituer les premières collections d'histoire naturelle du futur Muséum de Grenoble. Véritable cabinet princier, le cabinet de curiosités des hospitaliers de Saint-Antoine renfermait des monnaies et des 
médailles, des antiques dont une momie de femme, deux vases canopes en albâtre, des amphores, des bronzes antiques. Il contenait aussi des naturalia - c'est-à-dire des spécimens d'histoire naturelle, autant de pièces d'art ou d'histoire naturelle que l'Ordre faisait remonter depuis leur commanderie de Marseille vers Grenoble en longeant le Rhône, ou en s'approvisionnant auprès de leurs maisons installées en Europe?

Sur le catalogue de la bibliothèque des Antonins que le docteur Gagnon a transportée également, on retrouve de façon émouvante son nom écrit de sa main en marge des références de certains ouvrages $^{8}$. Les livres auxquels il s'intéresse sont tous des traités scientifiques: des ouvrages de minéralogie et de conchyliologie (la science des coquilles), mais aussi des ouvrages d'égyptologie comme la première description de l'Égypte intitulée La Description de l'Égypte sur les mémoires de M. Maillet, faite par l'abbé Le Masenier en $1734^{9}$. L'égyptologie était en germe dans le cabinet de curiosités transmis par les Antonins, dans les objets égyptiens qu'ils léguèrent, mais aussi dans ce catalogue de leur bibliothèque. Le catalogue faisait état de l'intérêt de l'ordre pour son histoire et celle de saint Antoine l'Égyptien, anachorète de Thébaïde : la légende voulait en effet que les reliques du saint aient été ramenées de Constantinople en 1070 par Jocelin, seigneur dauphinois, à l'issue d'un pèlerinage en Terre sainte. Au fil du temps, les Antonins étaient devenus des savants. Ils s'étaient rendus célèbres en guérissant le mal des ardents, un fléau du Moyen Âge attribuable à l'ergot du seigle qui empoisonnait le sang et provoquait abcès et gangrène. Les chirurgiens antonins amputaient à la scie. Médecin et chirurgien comme les Antonins, Gagnon hérita d'eux également sa passion pour l'égyptologie. Il se fit passeur de savoir et transmit leur héritage au jeune Jean-François Champollion ${ }^{10}$, son protégé, son ami, alors bibliothécaire et garde avec son frère du Cabinet d'histoire naturelle de Grenoble ${ }^{\mathbf{n}}$.

Tous ces détails, toutes ces précisions, comme le nom de Gagnon inscrit en marge du catalogue de l'abbaye ou sa participation au transport du cabinet de curiosités, ont leur importance dans la création d'un musée et plus particulièrement dans la reconstitution du cabinet d'un érudit : ils sont le signe d'une appropriation d'un objet muséal par l'érudit, ils montrent que le savant ne faisait pas de séparation entre son univers de recherche et son
7

"Entre royaume de France et Saint-Empire: les maisons des Antonins dans le Centre-Est». In. MISCHLEWSKI, Adalbert. Un ordre hospitalier au Moyen Âge: les chanoines réguliers de Saint-Antoine-en-Viennois. Grenoble: Presses universitaires de Grenoble, 1995, p. [220], carte $n^{\circ} 4$ (La pierre et l'écrit).

8

BIBLIOTHËQUE DE GRENOBLE. Catalogue du médaillier des Antonins, Musei Antonioni, 1752-1761 (BMC, R 4743).

9 MAILLET, Benoit de (1656-1738): consul de France en Egypte sous Louis XIV, auteur de la Description de l'Egypte contenant plusieurs remarques sur la géographie ancienne et moderne de ce païs. Paris: Cenneau et Rollin, 1735 (BMC, D.131). La Description de l'Égypte dont le préfet Fourier rédigera la préface à Grenoble date, quant à elle, de 1809: FOURIER, Jean-Baptiste. Histoire, Egypte, description de l'Egypte, ou recueil des observations et des recherches qui ont été faites en Egypte pendant l'expédition de l'armée française, publié par les ordres de Sa Majesté l'Empereur Napoléon le Grand, première livraison, préface historique. Grenoble, Peyronard, 1809 (BMG, H 57) (vol. de textes BMC, A 1193; vol. de pl. BMC, H 125).

10

CHAMPOLLION, Jean-François (1790-1832): égyptologue français, élève au lycée de Grenoble; professeur adjoint d'histoire à la Faculté des lettres de l'Académie de Grenoble; nommé bibliothécaire adjoint en 1812; il élabora en 1811-1812 le premier état des spécimens d'histoire naturelle contenus dans les pièces égyptiennes du cabinet de curiosités des Antonins; il vint à Paris en 1807 pour y suivre les cours de l'École des langues orientales et du Collège de France; découvreur des hiéroglyphes en 1822 (découverte consacrée dans le célèbre document Lettre à Monsieur Dacier en 1822), titulaire en 1831 de la Chaire d'égyptologie créée pour lui au Collège de France. 


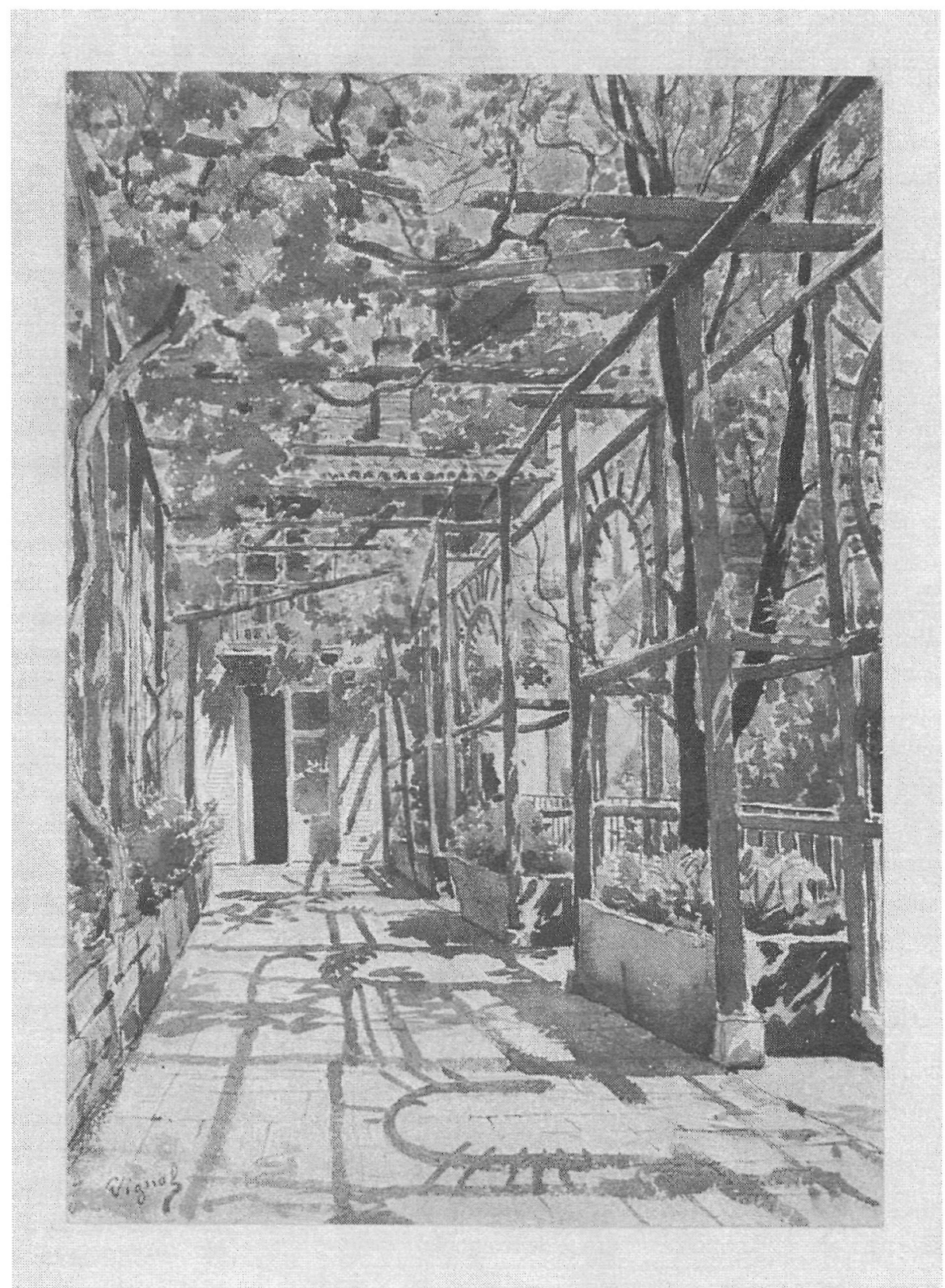

\section{Illustration 5}

Aquarelle de la terrasse par Pierre

Vignal, tiré de FAURE, Gabriel.

Pèlerinages dauphinois. Grenoble:

Éditions Jean Rey, 1920.

Collection bibliothèque de Grenoble, Vh. 190. 
univers personnel. Ils permettent alors à l'historien de déplacer le savant de son laboratoire pour le situer dans un autre univers, celui de son environnement familial. Cette osmose entre deux univers ou ce passage permanent d'un univers à l'autre dans la vie du personnage autorisent ainsi la reconstitution fictive. Et c'est bien la connaissance et l'étude d'un autre cabinet, le Cabinet d'histoire naturelle de Grenoble cette fois-ci, ancêtre du muséum actuel, qui nous a permis de reconstituer le cabinet personnel et privé du docteur Gagnon dans la maison où avait grandi Stendhal.

À la connaissance d'un personnage que l'historien perçoit du bout des doigts et que l'archiviste Arlette Farge nomme "la chair de l'archive " $"$, s'ajoute la lecture des grands textes. On retrouve le docteur Gagnon dans l'œuvre autobiographique de Stendhal Vie de Henri Brulard.Touche après touche, l'auteur évoque les goûts et les idées philosophiques du médecin, voltairien, à l'esprit ouvert et tolérant:

Dans les faits, j'ai été exclusivement élevé par mon excellent grand-père $M$. Henri Gagnon. Cet homme rare avait fait un pèlerinage à Ferney pour voir Voltaire et en avait été reçu avec distinction. Il avait un petit buste de Voltaire gros comme le poing [...] On disait toujours: $M$. de Voltaire, et mon grand-père ne prononçait ce nom qu'avec un sourire mélangé de respect et d'affection ${ }^{13}$.

"M. Henri Gagnon portait une perruque poudrée, ronde, à trois rangs de boucles, parce qu'il était docteur en médecine, et docteur à la mode parmi les dames, accusé même d'avoir été l'amant de plusieurs, entre autres madame Teisseire, l'une des plus jolies de la ville ${ }^{14}$." Comme nous le dépeint Stendhal, il faut imaginer le docteur Gagnon chez lui, dans son petit cabinet d'histoire naturelle, lisant les nouveaux titres de la bibliothèque en attendant ses malades : "J'épiais le moment où il était le plus occupé dans son fauteuil à lire les livres nouveaux dont, je ne sais comment, il avait toujours grande abondance ${ }^{15} . "$

Le petit-fils évoque maintes fois le grand-père sur sa terrasse, devant le cabinet d'histoire naturelle, arrosant ses fleurs, et l'intérêt mêlé que celui-ci portait aux lettres et aux sciences : "J'aidais toujours mon grand-père à arroser les fleurs, et il me parlait de Linné et de Pline, non pas par devoir mais par plaisir ${ }^{16} . »$ $n$

ROCHAS, Joëlle. "La Part de l'égyptologie dans les origines scientifiques du Muséum de Grenoble: les travaux des frères Champollion au Cabinet d'histoire naturelle de Grenoble (1809-1812)". In. Actes du IXe Congrès International des égyptologues, 6-12 septembre 2004, Grenoble-France. Louvain (Belgique): Peeters, 2007 p. 1611-1612 (Orientalia lovaniensia anlecta; 150).

12

"Ainsi naît le sentiment naïf, mais profond, de déchirer un voile, de traverser l'opacité du savoir et d'accéder, comme après un long voyage incertain, à l'essentiel des êtres et des choses. L'archive agit comme une mise à nu; ployés en quelques lignes, apparaissent non seulement l'inaccessible mais le vivant. Des morceaux de vérité à présent échoués s'étalent sous les yeux: aveuglants de netteté et de crédibilité. II n'y a pas de doute, la découverte de l'archive est une manne offerte justifiant pleinement son nom: source. " FARCE, Arlette. Le Goût de l'archive. Paris: Seuil, 1989. p. 14-15 (Points; Histoire).

13

STENDHAL. Vie de Henry Brulard. Paris: Honoré et Edouard Champion, 1913 , t. 1,

p. 34 et 113.

14

Id., p. 34-35.

15

Id., p. 191.

16

Id., p. 190 
17

DUCROS, père Etienne (1735 -1814):

franciscain, bibliothécaire et premier garde du Cabinet d'histoire naturelle de Grenoble de 1775 à 1808 ; académicien administrateur de l'Académie delphinale en 1775 ; bibliophile et excellent ornithologue, il fut en relation, notamment par sa riche correspondance, avec diverses personnalités scientifiques, dont le géologue Dolomieu, Faujas de Saint-Fond ou le botaniste Villars; il fut initié à la franc-maçonnerie à Grenoble en 1767. 18

Ibid., p. 214-215; à la p. 190.

19

STENDHAL, 1913, Vie de Henry Brulard, p. 55.

20

DEWACHTER, Michel . "L'Égypte ancienne dans les cabinetz de raretez du sud-est de la France aux XVIIe et XVIII siècles". In. Hommages à François Daumas, Montpellier: Université de Montpellier, Institut d'égyptologie, 1986, p. 184-189.
Tous ces détails puisés dans l'œuvre de l'écrivain seront précieux pour l'aménagement de la bibliothèque et du cabinet dans la maison Gagnon. Le docteur Gagnon fut aussi l'un des promoteurs de la bibliothèque de Grenoble, une bibliothèque pionnière puisqu'elle est, dans l'histoire des bibliothèques, la première bibliothèque publique en France. Les Dauphinois avaient en effet réuni une souscription afin de racheter la bibliothèque de leur évêque défunt $\mathrm{M}^{\mathrm{gr}}$ de Caulet, bibliothèque exceptionnelle, composée de 45000 volumes et convoitée par l'impératrice Catherine de Russie. À la suite du rachat de la bibliothèque, Gagnon proposa le père Ducros $^{17}$, chimiste et bibliophile, au poste de bibliothécaire et de premier garde du Cabinet d'histoire naturelle de Grenoble:

Le Père Ducros, ce cordelier que je suppose homme de génie, avait perdu sa santé en empaillant des oiseaux avec des poisons [...] Mon grand-père me parlait avec le même intérêt de l'Égypte, il me fit voir la momie achetée par son influence pour la Bibliothèque publique; là, l'excellent père Ducros (le premier homme supérieur auquel j'ai parlé de ma vie) eut mille complaisances pour moi $^{18}$.

L'amitié qui unit le docteur Gagnon et le père Ducros fut profitable aux institutions auxquelles ils collaborèrent: la bibliothèque de Grenoble et le Cabinet d'histoire naturelle. Ensemble les deux hommes influencèrent ou formèrent la nouvelle génération: Stendhal pour la littérature et Jean-François Champollion pour l'égyptologie.

Le docteur Gagnon, nous révèle Stendhal, avait fait ses études de médecine à Montpellier ${ }^{19}$. Cette ville, et d'une façon plus générale la plupart des grandes villes du Languedoc et de la Provence, recelaient aux XVII ${ }^{\mathrm{e}}$ et XVIII ${ }^{\mathrm{e}}$ siècles de nombreux cabinets de curiosités appelés alors cabinetz de raretez. Ceux-ci appartenaient à des évêques, des intendants, des magistrats, des chanoines, des médecins, des chirurgiens ou des apothicaires, tous en relation avec l'Égypte grâce aux gros négociants de Marseille, auxquels ils étaient par ailleurs souvent apparentés. Ce fut le cas notamment à Aix au XVII ${ }^{\mathrm{e}}$ siècle du cabinet de Peiresc, conseiller au Parlement de Provence, celui du président Bon à Montpellier au $\mathrm{XVIII}^{\mathrm{e}}$ siècle et celui d'Esprit Calvet, médecin à Avignon ${ }^{20}$. Nous conjecturons alors que c'est dans la ville de Montpellier, au cours de ses études de médecine, que Gagnon put se familiariser avec 
l'Égypte ancienne et former, développer son goût pour les antiquités. C'est là qu'il put nouer les premiers contacts - nous pensons notamment à Esprit Calvet qui deviendra d'ailleurs associé libre de l'Académie delphinale - ; c'est là qu'il put observer le jeu des relations et s'en inspirer pour reproduire le même schéma plus tard à Grenoble : «Mon grand-père me parlait avec le même intérêt de l'Égypte, il me fit voir la momie achetée, par son influence, pour la bibliothèque publique ${ }^{21}$."

Les médecins formés à Montpellier furent en contact avec des Languedociens ou des Provençaux établis en Orient. Ils favorisèrent dès le XVII ${ }^{\mathrm{e}}$ siècle puis tout au long du XVIII ${ }^{\mathrm{e}}$ les liens entre la ville et l'Égypte. Ils permirent la circulation de l'apothicairerie, mais aussi des antiquités égyptiennes pour l'approvisionnement des cabinets de raretés du sud-est. Il n'est donc pas étonnant de voir figurer, quelque temps après les années de formation du docteur Gagnon, le nom du docteur Calvet parmi les associés libres de l'Académie delphinale. On peut y lire également le nom de Mure, un cousin du docteur Gagnon, consul général de France en Égypte à Alexandrie, de 1774 à 1790 . Il est ainsi permis d'observer, entre Grenoble et l'Égypte, un jeu relationnel similaire à celui développé aux XVII ${ }^{\mathrm{e}}$ et XVIII ${ }^{\mathrm{e}}$ siècles entre les villes de Provence et du Languedoc et l'Égypte. Le consul Mure fit don en 1779 à la bibliothèque publique de Grenoble d'objets égyptiens : une momie et des vases canopes. Mais on trouve, dans la comptabilité du cabinet à la date de 1781, la trace d'un autre envoi vraisemblablement effectué par le consul Mure: une caisse venant d'Égypte et contenant "des oiseaux et des pétrifications". Ces spécimens égyptiens d'histoire naturelle rejoignirent les collections du Cabinet d'histoire naturelle de Grenoble. Bon nombre d'entre eux ont été obtenus grâce aux contacts du docteur Gagnon.

Le docteur Gagnon fut un personnage central sur lequel convergèrent tous les chemins conduisant à l'institution scientifique nouvellement créée: le Cabinet d'histoire naturelle de Grenoble. On retrouve chez lui l'ensemble des traits qui caractérisèrent le Cabinet d'histoire naturelle puis le Muséum de Grenoble: un goût prononcé pour l'égyptologie naissante, l'amour du Dauphiné concrétisé par la constitution de collections dauphinoises, l'attrait pour les collections exotiques. L'exercice de sa profession l'introduisit dans toutes les couches de la population grenobloise,
STENDHAL. 1913. Vie de Henry Brulard, p. 190. 


\section{Illustration 7}

Quartz.Illustration de la collection minéralogique du Cabinet d'histoire naturelle de Grenoble, XVIII'-XIX siècles. Collection du Muséum d'histoire naturelle de Grenoble

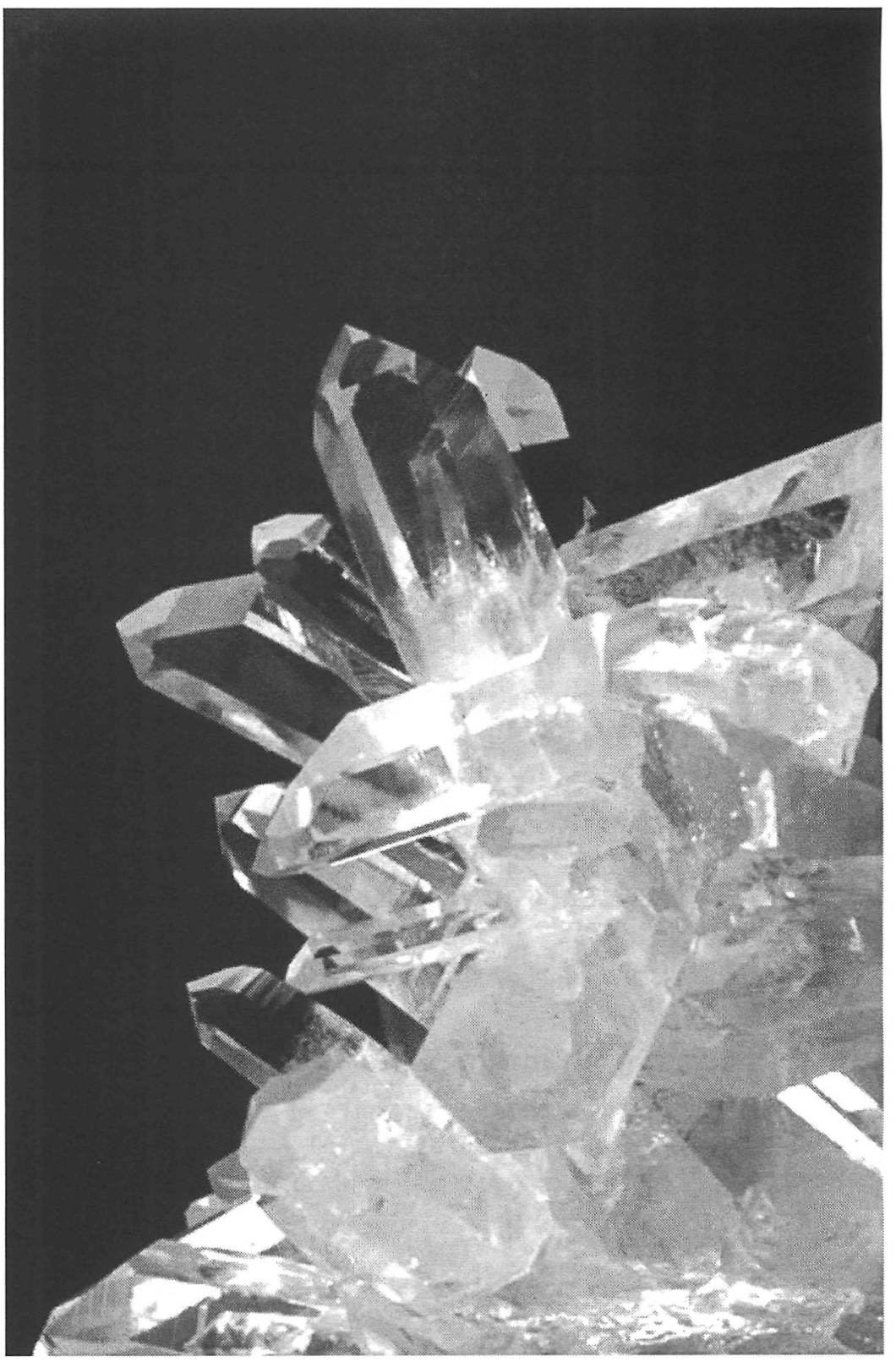




\section{Illustration 8}

Crocodile du Nil.

Collection du Muséum d'histoire

naturelle de Grenoble

\section{Illustration 6}

Coquille illustrant la collection conchyliologique du cabinet de curiosités de Raby l'Américain.

Collection du Muséum d'histoire

naturelle de Grenoble
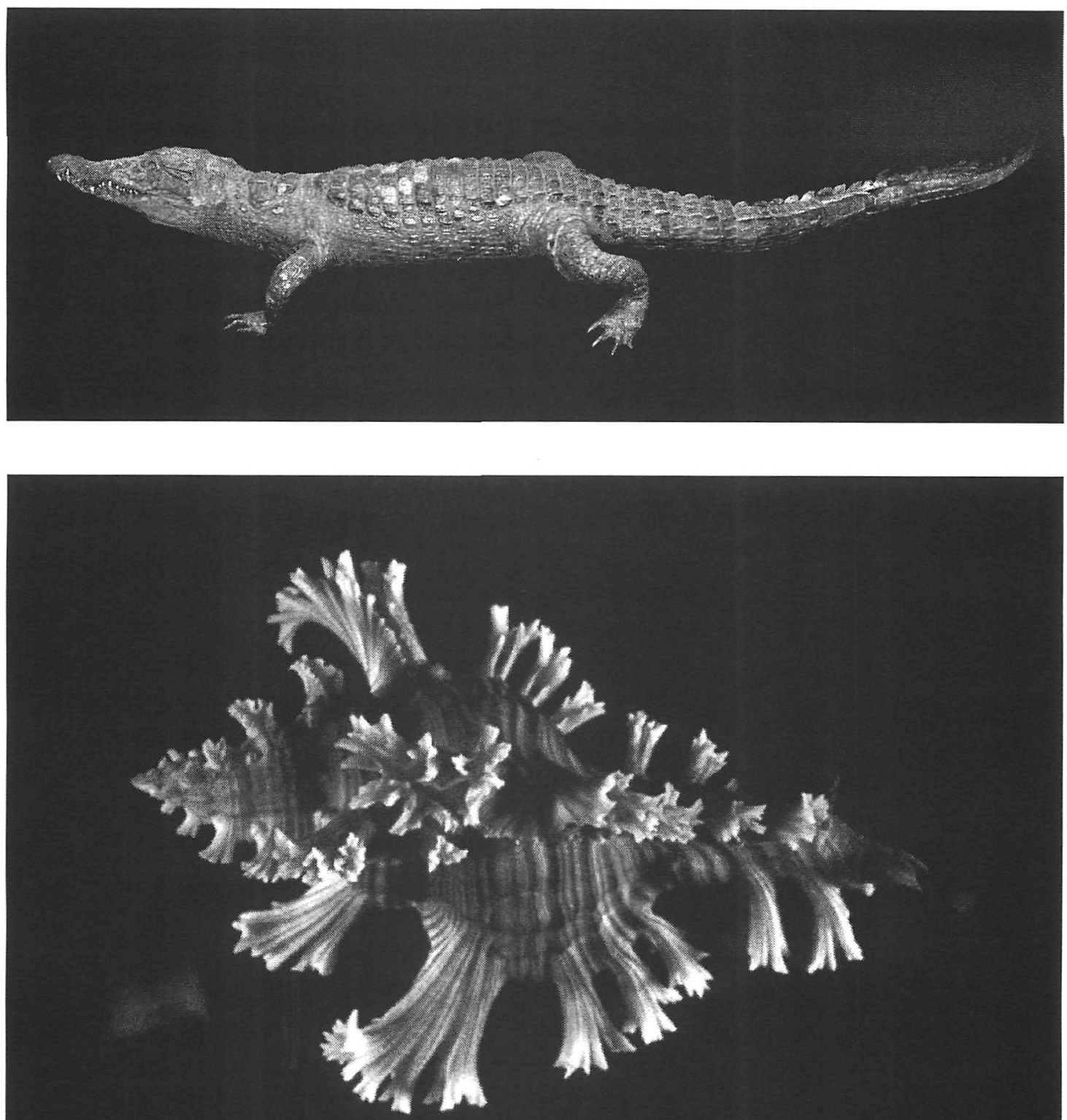
22

ROCHAS, Muséum de Grenoble: une histoire naturelle, op. cit.

23

BUFFON, Georges Louis Leclerc, comte de (1707-1788): naturaliste et écrivain français, il entra à l'Académie des sciences en 1733 et fut nommé intendant du Jardin du roi (futur Muséum national) en 1739; auteur d'une Histoire naturelle, en 36 volumes (1749-1804).

\section{4}

DEZALLIER D'ARGENVILLE, Antoine Joseph. "Des plus fameux cabinets d'histoire naturelle qui sont en Europe ". In. La Conchyliologie. Paris: De Bure, 1780, chap. IX (BMG, A 2717). de la noblesse parlementaire aux négociants et aux boutiquiers. Son érudition et son charme le faisaient apprécier dans les différents salons grenoblois, dans lesquels il lui arriva d'ailleurs de rencontrer l'élégant Romain Gagnon, un libertin, son fils. Ce dernier lui fit connaître à son tour le milieu maçonnique. La fréquentation des salons aristocrates donna à Gagnon un goût princier déjà bien développé par l'amour de l'Antiquité et des belles-lettres. Dans les projets auxquels il participa, il ne fit que des choix ambitieux, le but étant de se doter d'un établissement ou d'une collection exceptionnels, sans aucune considération de prix. Gagnon a marqué de son influence l'achat de la bibliothèque de $\mathrm{M}^{\mathrm{gr}}$ de Caulet, évêque de Grenoble. Au fil du temps, il a très certainement influencé la construction ambitieuse du Muséum d'histoire naturelle de Grenoble. Henri Gagnon sut faire profiter les savants grenoblois de ses différentes connaissances, si bien qu'il est permis d'observer que tous les chemins du Cabinet d'histoire naturelle de Grenoble se croisèrent et se recroisèrent inlassablement sur la personne de Gagnon.

\section{Cabinets de curiosités et cabinets d'histoire naturelle dans le mouvement des sciences}

Cinq cabinets nous ont aidée à recomposer le cabinet d'histoire naturelle du docteur Gagnon : trois cabinets de curiosités dauphinois et deux cabinets d'histoire naturelle $e^{22}$. Les trois cabinets de curiosités sont le cabinet des Antonins, celui de Raby l'Américain et celui du père Ducros. Le premier des deux cabinets d'histoire naturelle est le Cabinet du Roi réorganisé par Buffon ${ }^{23}$ au milieu du XVIIIe siècle au Jardin du roi à Paris. Le second est le Cabinet d'histoire naturelle de Grenoble, recensé par le naturaliste Dezallier en 1780 parmi les plus beaux cabinets d'histoire naturelle en France et en Europe ${ }^{24}$.

Le docteur Gagnon connaissait bien les trois cabinets de curiosités, soit pour s'en être occupé personnellement, soit parce qu'ils étaient les cabinets de ses amis : le cabinet de curiosités des Antonins, celui du père Ducros et celui de leur ami commun, le négociant Raby l'Américain. Ces trois cabinets entreront par la suite en dons dans les collections du Cabinet d'histoire naturelle de Grenoble. Pour le cabinet de Raby, Gagnon et Ducros étaient 
présents lors de l'inventaire après décès de leur ami Raby et ce sont eux qui ont transporté les collections au Cabinet d'histoire naturelle de Grenoble. Gagnon avait pu largement s'inspirer de ce cabinet pour installer le sien. Bien que plus réduites, les collections qu'il avait rassemblées devaient ressembler à celles de ses amis. D'ailleurs, nombre des spécimens compris dans ses collections devaient être ceux offerts par ses amis naturalistes : on pense notamment à des minéraux des Alpes ou à quelques coquillages exotiques offerts par Raby, au retour de ses lointains voyages. Joseph Raby était surnommé à Grenoble Raby l'Américain ou encore Raby d'Amérique. Comme ses frères, il avait possédé des plantations à Saint-Domingue et, depuis cette île, avait voyagé en Amérique (Canada, Mississipi, Nouvelle-Angleterre, Mexique, Martinique, Guadeloupe, Guyane française, Pérou) puis était rentré à Grenoble, sa fortune faite et ses bagages remplis de coraux, de perroquets et de pirogues d'Indiens. Il avait également voyagé pour ses affaires à Londres et en Hollande, puis dans les Alpes (Suisse, Savoie, Piémont), à la recherche de minéraux pour son cabinet de curiosités. Parmi les spécimens offerts au père Ducros par ses amis, on pense aux médailles en plâtre que sculptait le père Ducros. Car ce dernier, comme tous les collectionneurs amis de Gagnon, ne collectionnait pas seulement les minéraux, les oiseaux naturalisés ou les livres. Il était également peintre et sculpteur. Dans ses moments de loisirs, il moulait de petits médaillons en plâtre représentant les hommes illustres de toutes les époques qu'il offrait à son entourage. Quelques exsiccatae, plantes et fleurs que lui aurait remises son ami le botaniste Dominique Villars, ont pu également achever de sécher à l'intérieur d'un herbier de plantes alpines ${ }^{25}$.Enfin, le docteur a pu exposer dans son cabinet, afin de les admirer avec ses amis mais aussi de les étudier, quelques spécimens du cabinet de curiosités des Antonins, des oiseaux d'Afrique et d'Amérique du Sud pour leurs jolies couleurs ${ }^{26}$, mais aussi d'énigmatiques pièces égyptiennes ${ }^{27}$. On reconnaît bien dans ce descriptif ce qui rattache encore ce cabinet aux antiques cabinets de curiosités : le goût mêlé pour les arts et les sciences sans aucune restriction, la bigarrure des collections, la représentation du monde en un petit théâtre au centre duquel vit le collectionneur, un petit théâtre ordonné par et pour le collectionneur ${ }^{28}$.

Mais le modèle à suivre en cette fin de XVIII ${ }^{\mathrm{e}}$ siècle est Buffon et nombre de collections appartenant aux cabinets de curiosités
25

VILLARS, Dominique (1745-1814): botaniste dauphinois à la renommée internationale, médecin à l'hôpital militaire de Grenoble (la botanique était alors la base essentielle de la thérapeutique), professeur de botanique à l'École centrale de Grenoble en 1782, administrateur de l'Académie delphinale en 1786; auteur de l'Histoire des plantes de Dauphiné (1786-1789); ami de Gagnon, du père Ducros et des frères Champollion; membre de 26 sociétés savantes en France et à l'étranger, il fut en relation avec les plus éminents représentants de la communauté scientifique de son époque; il installa à Grenoble l'appartenance à la tradition linnéenne; en 1805 , il quitta Grenoble pour occuper un poste de professeur à la Faculté de médecine de Strasbourg dont il devint le doyen. 26

Le catalogue ornithologique du cabinet de curiosités des Antonins faisait notamment mention d'un barbu de Ceylan, d'un jacanard et d'un tyran du Brésil, d'un cardinal huppé du cap de Bonne-Espérance, d'une perruche et d'un pic du Sénégal, d'un bengali, d'oiseaux-mouches ainsi que de colibris (premier Catalogue ornithologique exotique, Muséum d'histoire naturelle de Grenoble / MHNG). 27 Entre 1752 et 1761 , le cabinet de curiosités des Antonins avait renfermé 5400 monnaies et médailles, 360 antiques, dont une momie de femme, deux vases canopes en albâtre, des amphores, des bronzes antiques et des naturalia (spécimens d'histoire naturelle).

28

Précisons ici que le Traktat Inscriptiones vel Tituli Theatri Amplissimi (1565) de Samuel Quiccheberg, conseiller auprès du duc Albert $V$ de Bavière, est considéré comme le premier traité de muséologie au monde. La première traduction française intégrale du traité a été réalisée par Nicolette Brout et publiée en 2004 dans le catalogue de l'exposition L'extraordinaire jardin de la mémoire qui s'est tenue en 2004 en Belgique au Musée royal de Mariemont. 


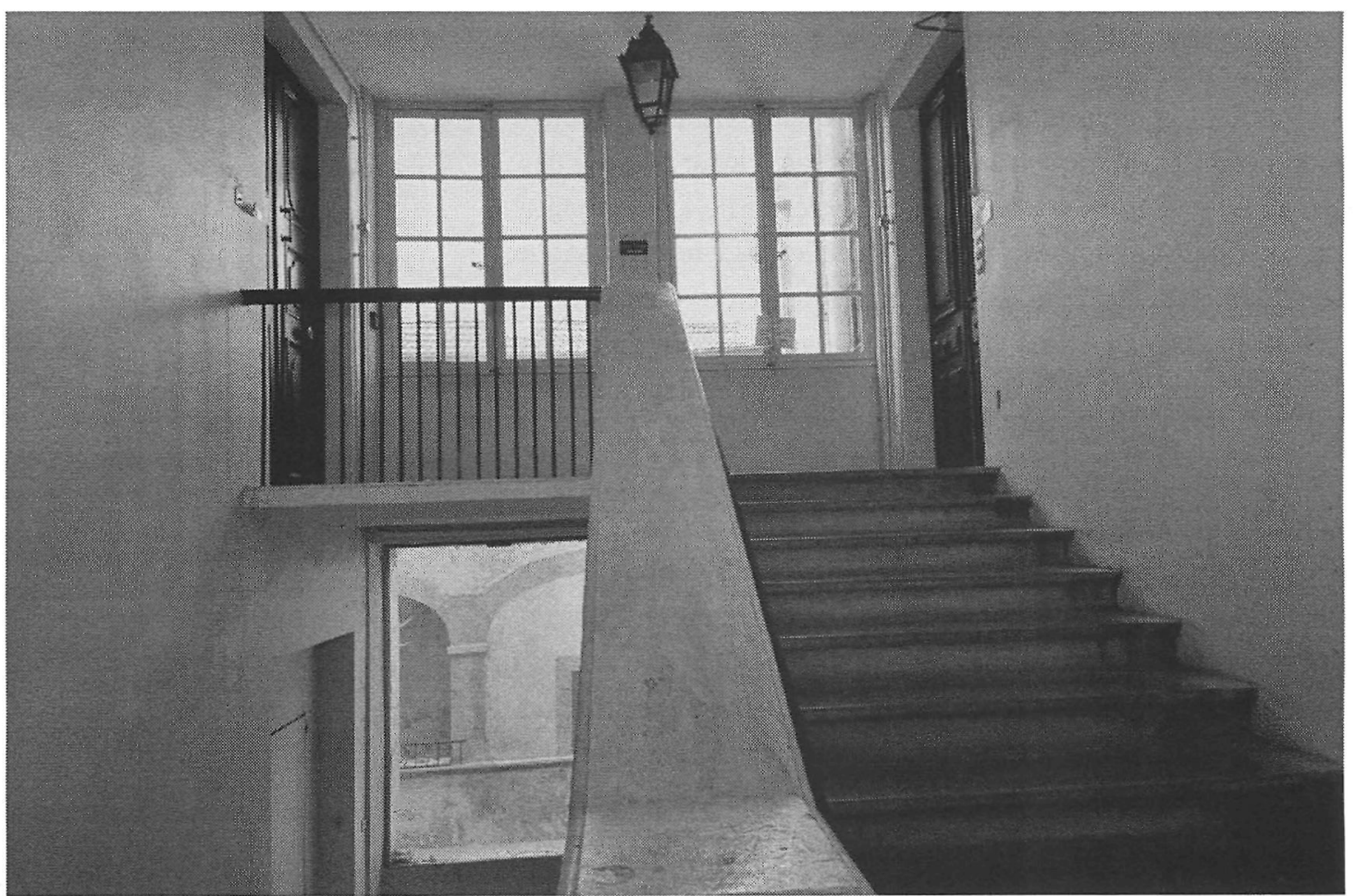

Illustration 9

"Grand escalier» de la maison Gagnon, inscrit en 2000 à l'Inventaire supplémentaire des monuments historiques.

Photo: Comité scientifique Musée Stendhal. 


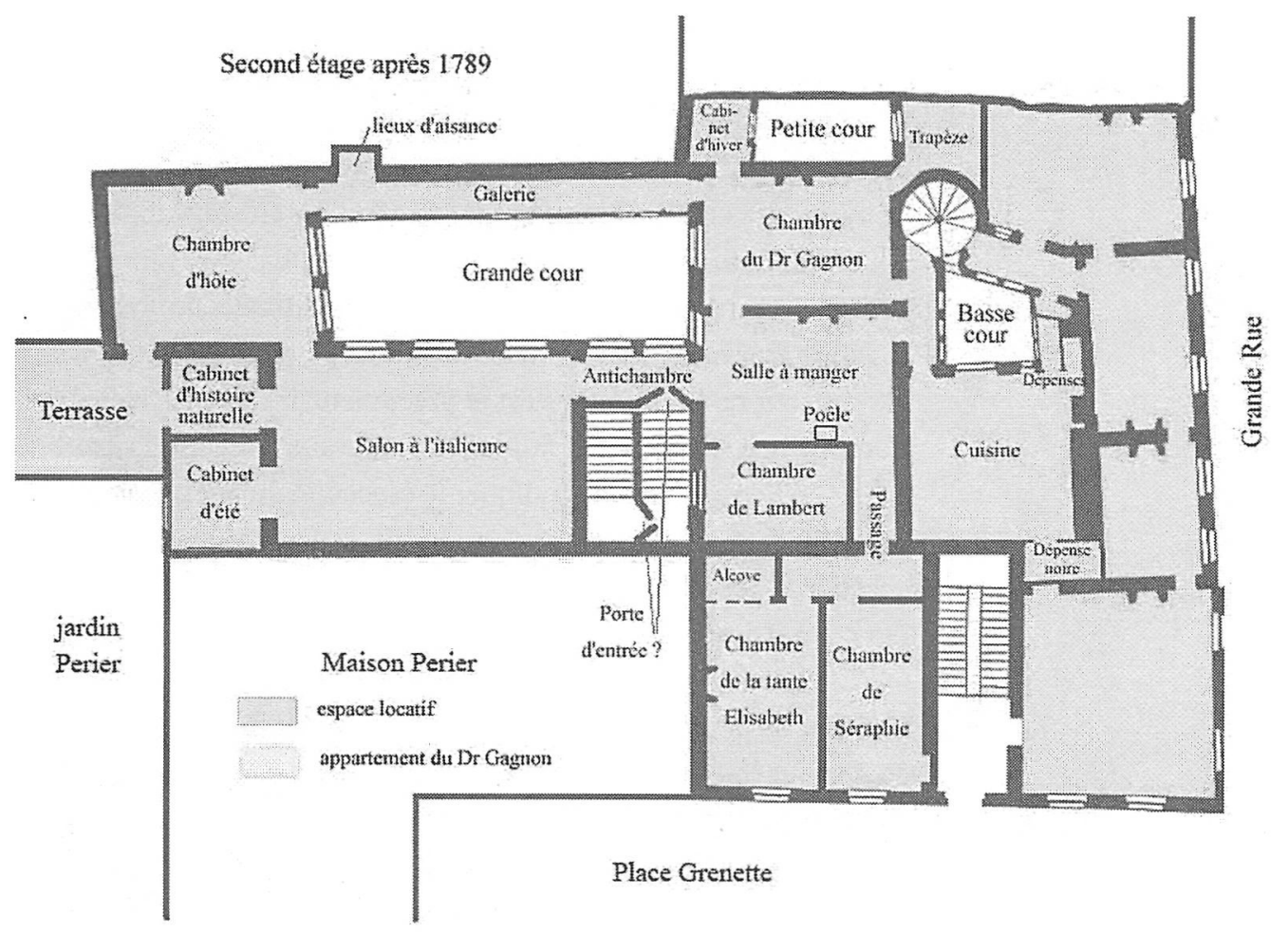

Illustration 10

Plan du second étage de la maison Gagnon avec indication du cabinet d'histoire naturelle et de la terrasse, d'après les croquis de Stendhal dans Vie de Henry Brulard.

Plan transmis par Gérald Rannaud, membre du comité scientifique du Musée Stendhal. 
29

Expression empruntée à POMIAN, Krzystof. Collectionneurs, amateurs et curieux: Paris, Venise, XVI-XVIII' siècle. Paris: Gallimard, 1987, 364 p.

30

Lire à ce propos les articles publiés sur Internet concernant le site européen dédié aux cabinets de curiosités: <http.//curiositas.org> (Universités de Poitiers et de Varsovie).
- notamment les artefacts des collections archéologiques, les collections anthropologiques ou les collections égyptiennes - vont disparaître, pour ne pas être conformes au nouveau classement en trois règnes exclusivement : minéral, végétal et animal, et sans qu'aucune perméabilité ne soit plus permise entre les arts et les sciences. À Grenoble, ces collections vont être reléguées dans un coin, avant d'être redécouvertes par le jeune Champollion, un autre protégé du docteur Gagnon.

Ces observations nous permettent de dégager, pour Grenoble, un type de cabinet assez spécifique, qui n'est plus un cabinet de curiosités et qui n'arrive pas à être pour autant un cabinet d'histoire naturelle, mais qui amorce déjà la phase suivante dans la métamorphose des cabinets : celle où les cabinets annoncent la venue du "muséum ". Ce nouveau concept, dont Grenoble avec ses collections égyptiennes est le précurseur, intègre les collections anthropologiques et fait la part belle aux collections exotiques. C'est ce que semble indiquer le crocodile du Nil pendu à la voûte du cabinet du docteur Gagnon (ill. 8). Invitation au voyage, obsédante présence de l'Égypte à Grenoble... Incontournable Égypte qui empêche à Grenoble tout "dressage de la curiositée ${ }^{29}$.

L'intérêt de la recomposition du petit cabinet d'histoire naturelle du docteur Gagnon est alors toute scientifique: dans les recherches qui sont aujourd'hui conduites en Europe (notamment en France, en Autriche, en Allemagne, en Pologne et en Italie) afin de donner une chronologie précise aux différentes étapes de la métamorphose des cabinets vers le muséum, le cabinet grenoblois avec sa spécificité égyptienne représente certainement un maillon des plus intéressants ${ }^{30}$.

\section{Vers le Musée Stendhal}

Nous résumons ici par un historique les différentes étapes de labellisations et de restaurations qui ont eu lieu depuis 2000 :

- 2000, inscription à l'Inventaire supplémentaire des monuments historiques portant sur l'appartement Gagnon $\left(150 \mathrm{~m}^{2}\right)$, l'ensemble des façades et des toitures, des allées et des galeries, ainsi que le grand escalier et l'escalier à vis (ill. 9) de la première cour; 
- 2002, aménagement en lieu d'animation littéraire de l'appartement natal de Stendhal, qui constitue le premier volet de restauration du patrimoine stendhalien;

- 2003, obtention du label Musée de France pour la collection du Musée Stendhal (peintures, dessins, photographies, en tout 1165 pièces appartenant à la Ville de Grenoble) conservée dans les réserves de la Bibliothèque municipale d'étude et d'information;

- 2006, acquisition du « Journal » de Stendhal par la Ville de Grenoble, venant ainsi compléter le fonds prestigieux conservé à la Bibliothèque ;

- 2008, lancement de l'étude de la partie nord du deuxième étage de la maison Gagnon pour concevoir un lieu muséal dédié à Stendhal ${ }^{31}$.

Le comité de pilotage du projet de Musée Stendhal est placé sous la présidence du maire de Grenoble représenté par son adjointe à la Culture. Il est composé des acteurs stendhaliens, patrimoniaux, des représentants de l'État, du département et de la région. Le comité scientifique chargé de développer le projet culturel se compose de trois professeurs de lettres spécialistes de Stendhal, d'un professeur de philosophie, d'une historienne des musées, de la directrice de la bibliothèque et du directeur du Muséum d'histoire naturelle.

\section{Le projet de Musée Stendhal est volontairement un projet éclaté} qui devra mettre en synergie les lieux et les collections existants dans un projet cohérent. Il comprend:

- Le fonds de livres et de manuscrits de Stendhal conservé à la bibliothèque: plus des trois quarts des manuscrits de Stendhal (Lucien Leuwen, Vie de Henry Brulard, etc.), soit parmi les plus importants fonds de manuscrits littéraires modernes, 7000 volumes imprimés (toutes les éditions originales et de nombreuses éditions en langues étrangères).

- La collection Musée Stendhal conservée à la bibliothèque (peintures, dessins, photographies, manuscrits, dont une partie sera exposée dans l'appartement de la maison Gagnon).

- L’appartement natal situé dans l'ancienne rue des Vieux-Jésuites où Stendhal a vécu jusqu'à la mort de sa mère.

- L'appartement Gagnon : la partie nord de l'appartement dans la maison Gagnon et dont la Ville dispose. C'était l'appartement officiel du grand-père Gagnon, celui dédié à la vie sociale et culturelle, voué à la réflexion intellectuelle. 


\section{Illustration 11}

Le cabinet d'histoire naturelle $\mathrm{du}$ docteur Gagnon dans un croquis de la Vie de Henry Brulard.

Bibliothèque de Grenoble

\section{Illustration 12}

Esquisse des armoires du cabinet d'histoire naturelle du docteur Gagnon. Musée Stendhal, phase de l'avant-projet définitif, 14 octobre 2008, Agence

Klapisch-Claisse. Échelle 1:50

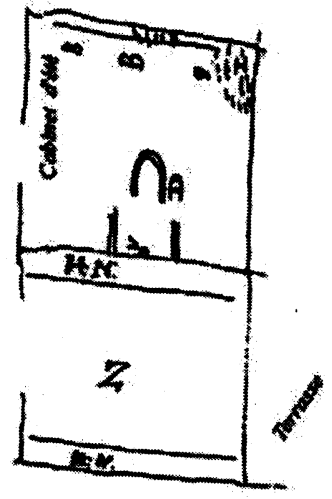

2 Cobber othoole motivelle AN mintrens olvesax

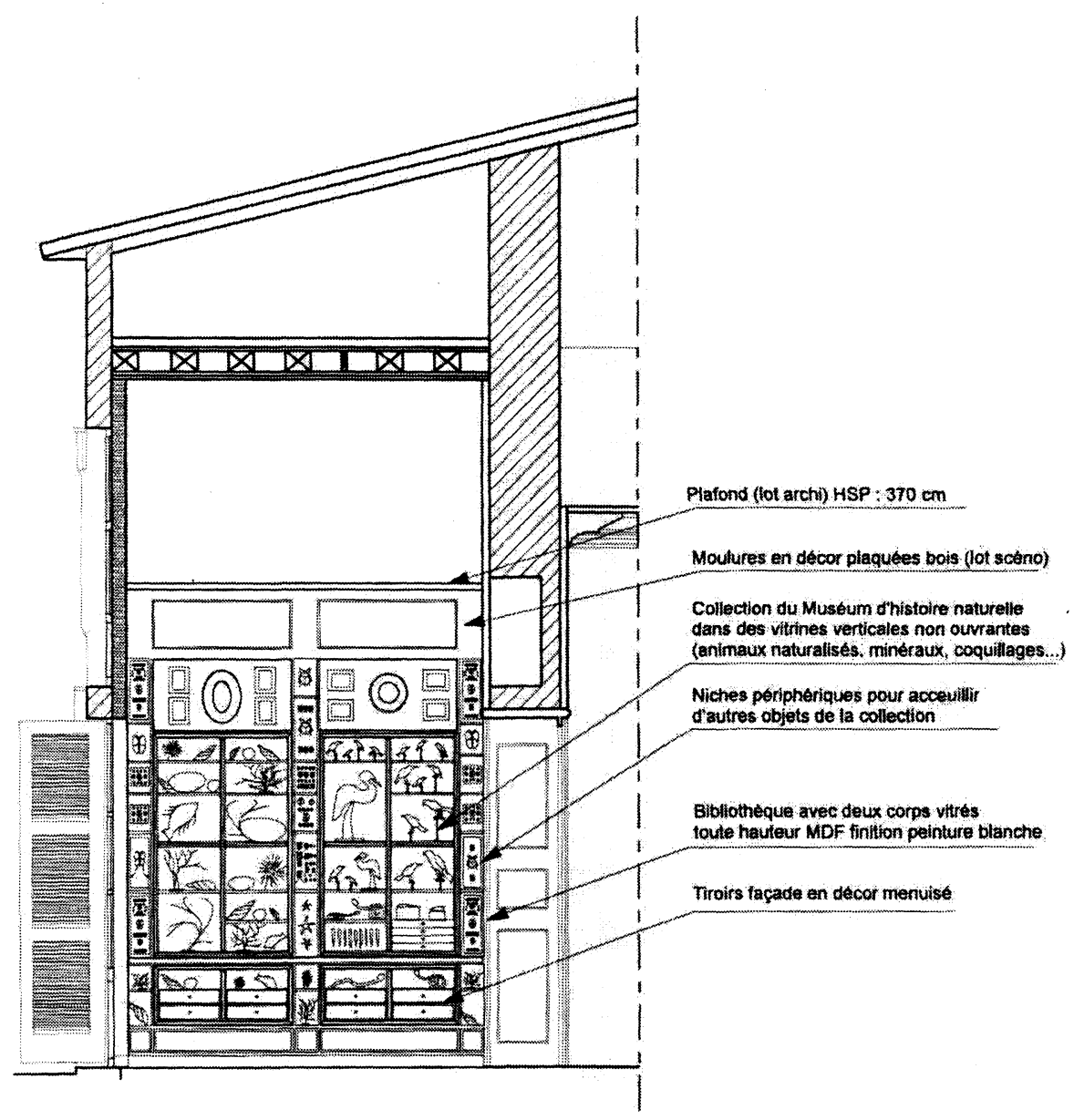


C'est là que Stendhal se réfugiait pour fuir sa tante et son père, et c'est là qu'il s'est formé intellectuellement jusqu'à seize ans au contact de son grand-père.

- Les parcours dans le centre ancien, enrichis et organisés par l'Office du Tourisme: certaines maisons ou certains lieux se rattachent intimement à la vie de Stendhal et sont décrits dans la Vie de Henry Brulard ${ }^{32}$.

- Les expositions temporaires : la programmation d'expositions prévoit une exposition conséquente à diffusion européenne tous les deux ans. Deux thèmes sont déjà à l'étude: "Stendhal l'Européen" en 2011 et "Stendhal et Rousseau, destins croisés " en $2012^{33}$.

Stendhal avait gardé un très agréable souvenir de la maison de son grand-père au cœur de la vieille ville, place Grenette, "située dans la plus belle position de la ville". Cette maison comprenait notamment l'ensemble du deuxième étage dont la terrasse remarquable (la fameuse "treille de Stendhal») est tournée vers le jardin de ville. Donnant d'un côté sur une cour intérieure du XVI ${ }^{\mathrm{e}}$ siècle et de l'autre sur le jardin de ville, le site actuel appartenant à la ville comprend quatre pièces. Deux espaces assez vastes et très bien éclairés : le grand salon à l'italienne sur lequel s'ouvre l'entrée de l'appartement; et la chambre d'hôte dite "de Romain Gagnon " (celle de l'oncle de Stendhal lorsqu'il visitait son père). Le grand salon est complété par deux petites pièces dont l'une donne sur la terrasse et la treille: le cabinet d'histoire naturelle du grand-père Gagnon, pièce typique de la bourgeoisie et de l'aristocratie éclairées de la fin du XVIII ${ }^{e}$ siècle; et le cabinet d'été (ou cabinet de travail), petite pièce de même surface à côté du cabinet d'histoire naturelle et consacrée à l'étude et à la lecture.

On découvre, à travers la disposition des pièces conçues par le docteur Gagnon, la vie des familles aisées de Grenoble au $\mathrm{XVIII}^{\mathrm{e}}$ siècle et cette vitalité intellectuelle qui a fait de Stendhal un héritier des Lumières (ill. 10).

Mais l'appartement aujourd'hui a bien changé. Le grand salon à l'italienne, autrefois magnifique, a été particulièrement malmené architecturalement et est actuellement tronqué par une cloison. Son plafond a de surcroît été surbaissé pour accueillir à mi-hauteur un autre étage qui casse le volume. D'autres aménagements faits 33 Précisons que Jean-Jacques se cachait à Grenoble en 1767, tout près de la maison Gagnon, sous le nom de Renou. II noua des liens avec des médecins et des herboristes connus de Gagnon. La maison des Charmettes dans laquelle il avait vécu avec $M^{\text {me }}$ de Warens de 1736 à 1742 en Savoie a été transformée en musée par la Ville de Chambéry (Musée des Charmettes, qui comprend également un jardin botanique reconstitué). 


\section{4}

HACHE, Jean-François dit Hache fils (1730-1796): ébéniste grenoblois issu d'une véritable dynastie de menuisiers et d'ébénistes.

35

"La maison se composait d'une cuisine à l'entresol, d'une salle à manger, d'une grande salle meublée avec goût - meubles en noyer, certains recouverts de marbre de la Porte de France, tables en bois doré et en marqueterie, un trumeau au-dessus de la cheminée et deux grandes glaces ornant les murs, fauteuils recouverts de tapisserie, des lampes, un lustre, une pendule, des tableaux - donnant sur le jardin, et d'une antichambre où l'on rangeait le linge de maison 'à la Venise'. La quantité de linge de maison -70 paires de draps, 85 douzaines de serviettes, 107 nappes de Venise - les quantités impressionnantes de vaisselles contenues dans les placards - seize douzaines d'assiettes de faïence de Strasbourg et de porcelaine -, la somptuosité de l'argenterie, les écuelles en or et en argent, les nombreuses cafetières, les services de verres et les carafes sont autant de marques de la richesse du maître de maison et du train de vie mené par Raby l'Américain. " Description de la maison de Raby l'Américain, dans la thèse de Joëlle ROCHAS, Université de Grenoble, 2006, d'après l'Inventaire de la succession de Raby. Grenoble, mars 1781 (Archives départementales de l'Isère, Grenoble/ADI, 2 E 704).

36

MUSEEE STENDHAL. Comité scientifique. Projet d'ordre du jour, op. cit., p. $34-35$. au cours du XXe siècle lui donnent aujourd'hui un aspect pitoyable. Il faudra par conséquent redonner de la respiration et du prestige à cette salle et éviter de la saturer par une muséographie trop lourde. Le grand salon était autrefois la pièce de réception. Il devait être finement décoré : rideaux, mobilier $\mathrm{Hache}^{34}$, linge de Venise, vaisselle précieuse, comme quelques rues plus loin, chez Raby l'Américain, l'ami du docteur Gagnon ${ }^{35}$. Le docteur Gagnon y recevait ses amis, les savants et les naturalistes. C'est là qu'on avait installé pour Stendhal une petite table de travail en face d'une des grandes fenêtres du salon qui donnait sur la grande cour. Du salon on passait dans le cabinet d'histoire naturelle où le docteur présentait les nouveaux spécimens de ses collections. La visite se terminait sur la terrasse pour admirer les dernières lueurs du soleil couchant sur le jardin Lesdiguières.

L'archéologie verticale pratiquée dans le grand salon à l'italienne a montré que les murs étaient recouverts au XVIII ${ }^{\mathrm{e}}$ siècle de papier peint dans des tonalités de rouge " pompéien ", avec des motifs que les archéologues n'ont cependant pas pu définir en raison de la petite taille du fragment retrouvé. La restauration du salon sera donc entreprise dans cette tonalité de rouge pompéien. Sur les plinthes, les fragments sont de couleur gris foncé; la peinture sera refaite dans cette couleur. Le cabinet d'été conserve dans un placard des fragments de papier aujourd'hui de couleur presque bleu de Prusse. Ce papier est cependant très encrassé, étant resté longtemps non recouvert, ce qui permet d'opter pour un bleu plus céruléen, un bleu moins pur, comportant une nuance de vert. Enfin, on a retrouvé dans le cabinet d'histoire naturelle des fragments de papier de couleur jaune de Naples avec un filet horizontal bleu de cobalt, à environ 20 centimètres de la plinthe basse ${ }^{36}$. Ce sont ces couleurs qui seront à nouveau appliquées dans le cabinet. Hélas, pour une meilleure déambulation et assurer la sécurité des visiteurs, un des murs du cabinet sera percé d'une porte, ce qui diminuera la taille des armoires du cabinet et le nombre de ses collections.

En raison du lieu de représentation qu'avait été le grand salon à l'italienne du temps du docteur Gagnon, le comité scientifique a décidé de lui conserver cette fonction de représentation et de le dédier au thème "Stendhal, la société, les hommes", permettant ainsi de présenter l'environnement familial et social de Stendhal. Voici les orientations qui ont été prises pour le grand salon: 
- affirmer le salon à l'italienne comme espace d'entrée et pièce centrale de l'appartement,

- retrouver sur l'ensemble du salon la hauteur du volume initial (environ 4,4 mètres),

- souligner les sens du parcours muséographique: du grand salon à l'italienne vers la terrasse,

- étudier la possibilité d'une sortie s'effectuant par l'extérieur à partir de la treille, en intégrant les contraintes liées à l'accès aux personnes à mobilité réduite,

- conserver des bureaux pour l'administration,

- utiliser des audioguides pour alléger la signalétique,

- aménager l'accueil.

Autant de contraintes indispensables pour transformer et aménager une maison d'écrivain en musée. L'intervention muséographique pour le grand salon prévoit un accrochage sur le grand mur du salon, organisé autour d'un buste et de deux tableaux représentant Stendhal. C'est ainsi Stendhal lui-même qui nous accueillera dans la maison de son grand-père.

L'idée de départ de dédier la chambre d'hôte, encore appelée "chambre de Romain Gagnon ", au thème du libertinage a finalement été abandonnée au profit d'une salle d'exposition temporaire du musée ${ }^{37}$. Cette idée sera cependant reprise lorsque la surface de l'appartement Gagnon sera plus étendue. On le voit, les orientations proposées pour l'approche muséographique ont été travaillées de façon à permettre une extension possible du musée. Elles ont également été conçues pour une autosuffisance de fonctionnement du musée. Dans l'attente d'une éventuelle extension, ces orientations permettent déjà de répondre au contexte historique, à l'œuvre littéraire de Stendhal, aux plans dessinés et commentés de la main de Stendhal, aux relevés actuels et aux anciens plans de l'appartement... et à l'impatience des amoureux de Stendhal.

\section{Les étapes de la reconstitution:} du cabinet idéal au cabinet réalisé

Les deux cabinets, cabinet d'été et cabinet d'histoire naturelle, associés à la treille, font partie d'un même ensemble dédié à l'étude. Du XVI ${ }^{\mathrm{e}}$ au XIX ${ }^{\mathrm{e}}$ siècle en effet, la bibliothèque du savant
37 Romain Gagnon était un libertin N'oublions pas que Grenoble avait servi de cadre au roman de Choderlos de Laclos Liaisons dangereuses et que Stendhal lui-même avait encore connu certains personnages qui avaient inspiré l'auteur pour son roman. 
38

ROCHAS, Joëlle. Les Cabinets d'histoire naturelle dans les appartements grenoblois du XVIII' et XIXe siècles: Raby, Ducros, Gagnon. Grenoble, 2 mai 2008 , résumé p. 9 (document remis au Comité scientifique et au scénographe). 39

Le rouge est la couleur dominante des spécimens d'histoire naturelle: coraux et oiseaux. La deuxième couleur qui domine chez les oiseaux est le vert. a toujours jouxté le laboratoire, lequel se prolongeait par l'expérimentation agronomique au jardin. L'historienne des sciences va cependant se concentrer sur le cabinet d'histoire naturelle.

Nous avons ainsi pu donner dans un premier temps la vision idéale d'un cabinet dans les appartements grenoblois du XVIII ${ }^{e}$ siècle, vision issue de nos travaux de recherche. Un premier document de huit pages à l'attention du comité scientifique portait sur les spécificités des cabinets des Antonins, ceux de Raby, de Ducros et de Buffon. Il décrivait le contenu d'une bibliothèque scientifique et donnait des précisions concernant les plantes cultivées ou acclimatées par les jardiniers collaborateurs de Dominique Villars. Il était accompagné d'un résumé en huit points destiné au scénographe ${ }^{38}$. C'est sur ce premier document, accompagné d'illustrations puisées dans des ouvrages de références, qu'a démarré la reconstitution. Le voici retranscrit dans sa partie "Résumé»:

1 Réaliser les armoires en bois qui composent le cabinet selon la description du Cabinet d'histoire naturelle de Grenoble en 1780.

2 Organiser les corps de rayonnages et les corps avec tiroirs comme chez Raby.

3 Sur les étagères, disposer une collection essentiellement minéralogique, alpine et exotique :

- pour la collection alpine, des minéraux de La Mure, d'Allemont et du Vivarais mais aussi des fossiles marins (dons probables des naturalistes et amis Ducros),

- pour la collection exotique, des coraux rouges (comme chez Raby).

4 Glisser ça et là, à la façon Raby, quelques pièces exotiques héritées des cabinets de curiosités: coquilles, oiseaux exotiques ${ }^{39}$.

5 Suspendre par une chaîne un crocodile empaillé au plafond.

6 On peut accrocher aux étagères des instruments scientifiques: instruments d'optique, lunettes astronomiques (comme chez Raby).

7 Accrocher aux murs du cabinet des reproductions des plâtres du père Ducros.

8 Ne pas oublier l'herbier dans la bibliothèque scientifique ni les ouvrages en relation avec les collections du cabinet et le jardin exotique d'expérimentation sur la terrasse. 
C'est lors de ces premiers échanges que nous avons pris conscience qu'historiens et scénographes n'utilisaient pas le même langage pour s'exprimer, les historiens fonctionnant avec l'écrit et les scénographes avec l'image. Pour bien se comprendre, de nombreuses images ont alors circulé avant que le choix ne se fixe sur un modèle de cabinet ${ }^{40}$.

Pour commencer, il est décidé de reprendre, pour la réalisation des armoires en bois du cabinet, la description faite par Ducros du Cabinet d'histoire naturelle de Grenoble: des corps de tablettes, tout en hauteur, à deux portes vitrées ${ }^{41}$. Cette description correspond également à celle que les manuscrits transmettent du cabinet de Raby, ainsi qu'au modèle d'un cabinet d'histoire naturelle donné par Buffon. En entrant dans le cabinet, le visiteur aura une vision exacte des trois ordres composant les sciences naturelles au XVIII ${ }^{\mathrm{e}}$ siècle : à gauche les minéraux, au centre les végétaux et à droite les armoires d'animaux.

À la demande des scénographes, deux nouvelles séries d'études ont été entreprises, la première concernant les couleurs et la seconde, à l'initiative du directeur du Muséum, concernant les odeurs. On y avait adjoint une étude sur les crocodiles et une sur les coraux rouges. On opte tout d'abord pour une peinture gris bleuté recouvrant les armoires, comme celle que l'on pouvait encore distinguer sur les vieilles armoires du Muséum de Grenoble en 1980 et qui correspond bien également aux armoires originales de Ducros. Cette couleur se rapproche de la couleur blanche du modèle érigé par Buffon pour l'installation d'un cabinet d'histoire naturelle au Jardin du roi.

Poursuivant notre étude sur les couleurs, notre collaboration avec les chercheurs autrichiens du Kunsthistorisches Museum de Vienne et notamment du Schloss Ambras près d'Innsbruck nous a permis d'établir que, à l'instar du cabinet de la bibliothèque Sainte-Geneviève dont il s'inspirait, le cabinet de curiosités des Antonins avait dû contenir des coffrets noirs et dorés au fond bleu, ce que Gagnon avait pu imiter ${ }^{A 2}$. (Précision supplémentaire: le fond des tiroirs contenant des petits spécimens ou des spécimens fragiles comme les cristaux était généralement recouvert de peaux de chamois, afin de le protéger contre les chocs et éviter
40 ROCHAS, Les Cabinets d'histoire naturelle dans les appartements grenoblois..., op. cit., résumé p. 9.

41 "Cinq corps de tablettes [en ébénisterie réalisée par Hache] à deux portes vitrées en verre de Bohême, avec crémaillères, serrures, peintes en gris et fermant à clef". In. Description du Cabinet d'histoire naturelle de Grenoble en 1780 , manuscrit issu de la comptabilité du père Ducros (MHNG).

42 SEIPEL, Wilfried. Die Entdeckung der Natur [La Découverte de la nature]. Vienne: Kunsthistorisches Museum, 2007, p. 31. [Trad. Joëlle Rochas]. 


\section{3}

La référence aux travaux autrichiens sur les cabinets de curiosités est due aux nombreuses influences reçues des Etats allemands par les institutions scientifiques dauphinoises au XVIII siècle. Lire à ce propos: ROCHAS, Joëlle. "L'Influence des naturalistes et des cabinets de curiosités germaniques dans la genèse du Cabinet d'histoire naturelle de Grenoble (17731839)" I, In. Histoire des Alpes = Storie delle Alpi $=$ Geschichte der Alpen, $n^{\circ} 14,2009$. 44

Id., p. 270-271. qu'il ne s'effrite à chaque ouverture du tiroir.) Mais, pour des raisons de protection des spécimens, il a été décidé que les armoires reconstituées ne contiendraient pas de tiroir amovible. Toujours pour permettre une meilleure conservation des spécimens exposés, l'éclairage des vitrines du cabinet sera extérieur et en lumière homogène. Pour les bocaux de fruits, on pourra en revanche envisager un éclairage intérieur placé à l'arrière. Précisons alors ici que le cabinet est idéalement orienté : nord-ouest. Comme dans les châteaux et les belles demeures, les bibliothèques et les cabinets d'histoire naturelle étaient situés dans des pièces à l'abri de la lumière et n'étaient jamais orientés plein sud. Le père Ducros protégeait les oiseaux de son cabinet en confectionnant pour les vitrines des rideaux faits dans de vieux draps. On ne tirait les rideaux pour ouvrir et observer les oiseaux que lorsqu'un visiteur s'annonçait. Ces précautions, qui ne peuvent plus convenir à la muséologie d'aujourd'hui, seront remplacées par une attention toute particulière accordée aux méfaits de la lumière ou des éclairages.

Un crocodile sera accroché au plafond du cabinet. Nous transmettons alors au directeur du Muséum, aux designers et aux membres du comité scientifique l'état des connaissances concernant les crocodiles des cabinets de curiosités et tel que mis à jour par les chercheurs autrichiens ${ }^{43}$. Deux types de crocodiles appendus ornaient les murs ou les plafonds des cabinets de curiosités : les crocodiles du Nil (les préférés des cabinets de curiosités); ou ceux que l'on appelle les "petits crocodiles ", qui sont la plupart du temps des alligators (les sauriens, quant à eux, viennent d'Afrique).

Nous avançons alors l'hypothèse que le docteur Gagnon, qui était en relation avec son cousin Mure, consul à Alexandrie, avait pu se procurer un crocodile du $\mathrm{Nil}^{44}$.

Les curieux des cabinets de curiosités étaient de grands amateurs de coraux. Les chercheurs autrichiens du Schloss Ambras d'Innsbruck relatent que c'est dans la mythologie que l'on trouve l'explication de la couleur rouge sombre des coraux chers aux cabinets de curiosités: quand Persée eut tranché la tête de la Méduse, le sang qui se répandit dans la mer se transforma en corail rouge. Le corail était aussi un matériau dont on se servait pour fabriquer des bijoux. On lui accordait un pouvoir magique : il enlevait le mauvais œil et aidait les enfants à mettre leurs premières dents; 
sous forme de poudre, il était un remède contre les maladies du sang et les menstrues. Dans les cabinets, les coraux noirs ou rouges étaient mis en exposition sur des socles de bois ${ }^{45}$. Autant de détails dont tiendront compte les scénographes ou qui serviront aux notices d'accompagnement.

Les cabinets d'histoire naturelle avaient une odeur dont on peut se faire une idée à la lecture des descriptions du cabinet de curiosités du père Ducros. C'était essentiellement l'odeur des produits que le père Ducros utilisait pour monter ses oiseaux en peaux: " de la poix, de la résine, du tabac, de l'alun pulvérisé, de la cire, des drogues en pots ", mais aussi de la colle et de la céruse pour la couleur ${ }^{46}$. Pour la conservation des animaux naturalisés, il utilisait de "l'esprit de térébenthine, du camphre et du souffre».Les fumigations d'huile de térébenthine étaient utilisées pour protéger les animaux naturalisés des insectes (teignes à fourreaux, anthrènes, attagènes, dermestes et ptilins). L'odeur très forte du camphre quant à elle repoussait les insectes. Utilisé en grandes quantités, ce camphre était un poison et c'est de ce poison utilisé à répétition que serait mort le père Ducros, nous dit Stendhal.

L'historienne et les naturalistes du Muséum d'histoire naturelle de Grenoble se sont enfin entendus sur les spécimens qui devaient peupler les armoires: herbier de plantes alpines ou de plantes exotiques acclimatées, bananes dans des bocaux, minéraux du Dauphiné et de l'ensemble de l'arc alpin, coquillages exotiques et coraux, oiseaux au plumage vif, sans oublier l'incontournable crocodile appendu. Quelques bagarres ont alors fusé sur l'introduction de serpents dans les collections du cabinet, serpents dont les catalogues ne font mention qu'à une période postérieure à la celle évoquée. Mais il faut bien être conciliante et concéder parfois quelque accroc à l'histoire.

Nous aimerions conclure sur la richesse du Musée Stendhal, sur l'originalité de son concept étendu, sur sa démarche muséale intéressante qui met en synergie plusieurs disciplines et dans laquelle nous avons reconnu l'homme Protée qu'était Henri Gagnon, un homme multidimensionnel qui s'intéressait à la fois aux lettres, aux sciences et aux arts.
46 Correspondance et comptabilité du père Ducros (MHNG). 
Translated by Sofia Arenzon
An Egyptologist's cabinet during the Enlightenment: Dr. Gagnon's natural history cabinet at the Stendhal Museum of Grenoble (France).

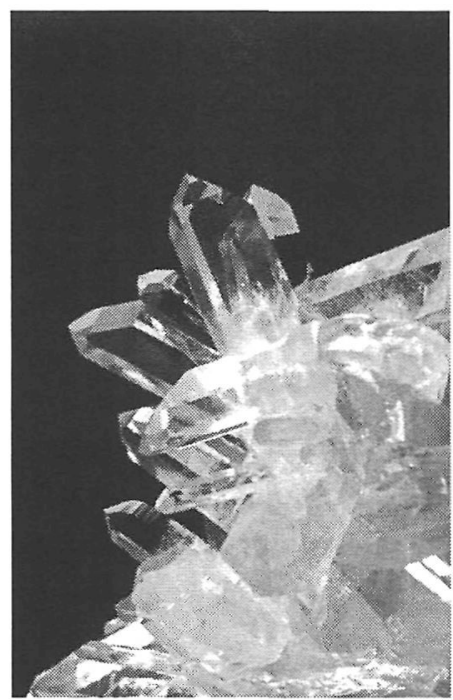

Quartz.Illustration de la collection minéralogique du Cabinet d'histoire naturelle de Grenoble, XVIII ${ }^{\text {eXIX }}{ }^{\mathrm{e}}$ siècles. Collection du Muséum d'histoire naturelle de Grenoble 
In 2008, Grenoble, a city located in the French Alps, has begun restoration work on the house of Dr. Gagnon, grandfather of Stendhal, a world-famous French writer. Dr. Gagnon, a true spirit of the Enlightenment, had a considerable influence on his grandson's education. Being a real stendhalian character, Dr. Gagnon is at the heart of Stendhal's autobiographic novel, The Life of Henry Brulard, in which the author recalls his first youthful years at his grandfather's house in Grenoble. Stendhal tells us that by the end of the $18^{\text {th }}$ century his grandfather has acquired a natural history cabinet at his house. There is even a plan of it in the book.

Being a science historian, a specialist of curiosity and natural history cabinets and having written a thesis on the Museum of Grenoble in 2006, I was approached by the Grenoble Office of Cultural Affairs to rebuild Dr. Gagnon's natural history cabinet at the Stendhal Museum. I was also chosen as a scientific expert for recreating Dr. Gagnon's terrace, an extension of the natural history cabinet, known in Grenoble as Stendhal's vine-arbour.The Gagnon House and the Stendhal Museum should open in September 2011, coinciding with the European Days of Heritage. The new museum will display a natural history cabinet and an experimental garden inside an 18th century house, next to a big Italian style drawing room and a library, dear to those who are passionate about natural history during the Enlightenment.
This article presents what was a natural history cabinet during the Enlightenment, while describing the various reconstruction phases in a writer's house. It differentiates between ideal and possible achievement, between scientific rigor and the requirements of a work master, between historic truth and museographic constraints. It remains a unique experience as this restoration testifies the extreme originality of Grenoble period cabinets. In fact, as the $18^{\text {th }}$ century saw an evolution of curiosity cabinets into natural history cabinets, Grenoble natural history cabinets were still very influenced by the former. People who made these collections had a passion for Egyptology and exotic collections. Their cabinets were far from being archaic but rather foreshadowed the forthcoming creation of museums. The reconstruction of Gagnon's natural history cabinet is the sole witness to the double identity of Grenoble cabinets. Their collections reflect among others an early passion for Egyptology and announce the arrival of young Champollion, an Egyptologist and friend of Dr Gagnon, future discoverer of hieroglyphs, one of the first curators of the future Museum of Grenoble and the first scientist to study Egyptian collections in the cabinets of the Dauphiné region. 